\begin{tabular}{|c|l|}
\hline Title & A nalytical regularization of hypersingular integral for Helmholtz equation in boundary element method \\
\hline Author(s) & Tomioka, Satoshi; Nishiy ama, Shusuke \\
\hline Citation & $\begin{array}{l}\text { Engineering A nalysis with Boundary Elements, 34(4), 393-404 } \\
\text { https://doi.org/40.1016/.enganabound.2009.10.011 }\end{array}$ \\
\hline Issue Date & 2010-04 \\
\hline Doc URL & http://hdl.handle.net/2115/42806 \\
\hline Type & article(author version) \\
\hline File Information & EABE34-4_393-404.pdf \\
\hline
\end{tabular}

Instructions for use 


\title{
Analytical Regularization of Hypersingular Integral for Helmholtz Equation in Boundary Element Method
}

\author{
Satoshi Tomioka ${ }^{\mathrm{a}, *}$, Shusuke Nishiyama ${ }^{\mathrm{a}}$ \\ ${ }^{a}$ Graduate School of Engineering, Hokkaido University, Kita 13 Nishi 8, Kita-ku, Sapporo, 060-8628 \\ Japan
}

\begin{abstract}
This paper presents a gradient field representation using an analytical regularization of a hypersingular boundary integral equation for a 2-dimensional time harmonic wave equation called the Helmholtz equation. The regularization is based on cancelation of the hyper-singularity by considering properties of hypersingular elements that are adjacent to a singular node. Advantages to this regularization include applicability to evaluate corner nodes, no limitation for element size, and reduced computational cost compared to other methods. To demonstrate capability and accuracy, regularization is estimated for a problem about plane wave propagation. As a result, it is found that even at a corner node the most significant error in the proposed method is due to truncation error of non-singular elements in discretization, and error from hypersingular elements is negligibly small.
\end{abstract}

Key words: Boundary Element Method (BEM), Helmholtz Equation, Hyper-singularity, Analytical Integral, Regularization, Gradient field, Error estimation

\footnotetext{
*Corresponding author: Tel.: +81-11-706-6656; Fax: +81-11-706-7128.

Email addresses: tom@qe.eng.hokudai .ac.jp (Satoshi Tomioka), shu@qe.eng.hokudai .ac.jp (Shusuke Nishiyama)
} 


\section{Introduction}

This paper presents a gradient field representation using an analytical regularization of a hypersingular boundary integral equation for a 2-dimensional time harmonic wave Helmholtz equation. This representation is applicable to avoid fictitious solutions which appear in an external problem such as a scattering problem. The fictitious solutions are only found at certain wave numbers which are corresponding to the eigenvalues of a related interior problem [1-6]. To avoid the fictitious solutions, various techniques have been presented thus far. At present, the most widely used techniques may be the techniques demonstrated by Burton and Miller [2]. They adopted a coupled two independent boundary integral equations. One is a conventional boundary integral equation (CBIE), in which the field value itself at a point on the boundary are shown as the boundary integral of which kernel includes fundamental solutions. The other is called a hypersingular boundary integral equation (HBIE) that derived by taking the normal derivative of the CBIE, in which the normal derivative or the gradient of the field are represented by the boundary integral. The integrand of HBIE includes the the second order derivative of the fundamental solution, which has stronger singularity than that of the CBIE.

An important feature of the HBIE integral is that it includes the second order derivatives of the fundamental solution. In case of CBIE, the singularity of the kernel is removed by analytical integral around singular point. However, in case of HBIE, since the singularity is stronger than that in CBIE, the hypersingular integral can not be evaluated without special considerations. Burton and Miller also proposed the double integral technique to regularize the hyper-singularity. This technique requires more intensive computational efforts because several computations of double integration are required. This paper attempts to achieve regularization without use of the double integral technique.

Previous methods to obtain regularization of hypersingular integrals for second order partial differential equation are reviewed by Tanaka et al. [7] and Chen et al. [8], and for the Helmholtz equation are summarized in literatures elsewhere; e.g., by Hwang [9], Yang [10], and Yan et al. [11]. The regularizations applied in past studies are 
classified into three approaches. The first is a use of tangential derivatives on the boundary $[12-14,19,20]$. The second approach is a use of the fundamental solution of the Laplace equation together with that of Helmholtz equation [2, 9-12, 16-18, 20]. Since the singularity of the fundamental solution of Helmholtz equation is same as that of the Laplace equation, the singularity of the difference of them becomes weakly singularity. The last scheme is a use of the difference between the field at an internal point and that on its corresponding boundary point $[9,14-18,20,25,26]$; most of these studies are used together with Taylor series expansion. The scheme presented in this paper applies these three schemes. Most of past studies using these techniques are applied for 3-dimensional problems and some can be applied curved surfaces; however, almost of them require a smooth boundary. Although this paper presents a scheme of regularization only for 2-dimensional problems, it is applicable for problems with corners.

After the regularizations, the singularity of a hypersingular integral becomes a weakly singular integral. However, to evaluate this integral properly some considerations are required to ensure accuracy; e.g., Meyer [14] and Chien [18] use sub-divided elements, Terai [15] has presented an analytical integral, and Yang [10] has used Fourier-Legendre expansions. This paper presents an analytical representation of hypersingular integral.

The outline of this paper is as follows. In section 2, the singularities of CBIE and HBIE are introduced. The regularization of the singularity of HBIE is presented in section 3. In section 4, a rough estimation of errors in integrals of both hypersingular elements and regular elements is presented. In section 5, numerical results are demonstrated for the D'alembert solution to show the error of a gradient field on the boundary in the HBIE representation. Finally, some remarks are shown in section 6.

\section{Representation of gradient field on boundary}

A time harmonic scalar wave $u(x)$ at a point $\boldsymbol{x}$ satisfies the following Helmholtz equation:

$$
\nabla^{2} u(\boldsymbol{x})+k^{2} u(\boldsymbol{x})=0, \quad \boldsymbol{x} \in \Omega,
$$


where $k$ indicates the wave number, and $\Omega$ represents the spatial domain considered. A fundamental solution $u^{*}(\boldsymbol{x} ; \boldsymbol{y})$ in free space corresponding to this equation satisfies

$$
\nabla^{2} u^{*}(\boldsymbol{x} ; \boldsymbol{y})+k^{2} u^{*}(\boldsymbol{x} ; \boldsymbol{y})=-\delta(\boldsymbol{x}-\boldsymbol{y}),
$$

where the differential operator $\nabla$ operates only on $\boldsymbol{x}$, but not on $\boldsymbol{y}$. Using Green's second identity and some integral operations, we can obtain the conventional boundary integral equation (CBIE),

$$
c(\boldsymbol{y}) u(\boldsymbol{y})=\oint_{\Gamma}\left[u^{*}(\boldsymbol{x} ; \boldsymbol{y})(\nabla u(\boldsymbol{x})) \cdot \boldsymbol{n}-u(\boldsymbol{x})\left(\nabla u^{*}(\boldsymbol{x} ; \boldsymbol{y})\right) \cdot \boldsymbol{n}\right] d \Gamma
$$

where $\Gamma$ denotes a boundary surrounding $\Omega, \boldsymbol{x}$ is the position of the points on the boundary, $\boldsymbol{y}$ is the position of a field point, $\boldsymbol{n}$ is the outward-pointing normal unit vector, and $c(\boldsymbol{y})$ is the result of the following evaluation of Dirac's delta function.

$$
c(\boldsymbol{y}) u(\boldsymbol{y}) \triangleq \int_{\Omega} u(\boldsymbol{x}) \delta(\boldsymbol{x}-\boldsymbol{y}) d \Omega=\int_{\Omega} \delta(\boldsymbol{x}-\boldsymbol{y}) d \Omega u(\boldsymbol{y}) .
$$

The coefficient $c(y)$ depends on both the relative position of field point $\boldsymbol{y}$ and the shape of boundary $\Gamma$. When $\boldsymbol{y}$ is located inside and outside the domain, $c(\boldsymbol{y})$ evaluates to 1 and 0 , respectively. In the case where $\boldsymbol{y}$ is located on the boundary, $c(y)$ equals to the ratio of interior angle $\Delta \theta$ to a whole angle; e.g., $\Delta \theta / 2 \pi$ for 2-dimensional problems.

A 2-dimensional fundamental solution appearing in Eq. (3) is written as a function of the distance between the source point (i.e., integration point $\boldsymbol{x}$ ) and the field point $\boldsymbol{y}$ [21],

$$
u^{*}(\boldsymbol{x} ; \boldsymbol{y})=\frac{1}{4 j} H_{0}^{(2)}(k r), \quad r=|\boldsymbol{x}-\boldsymbol{y}|
$$

where $j$ denotes an imaginary unit, and the function $H_{0}^{(2)}(k r)$ is a second kind 0 -th order Hankel function. This solution represents an outward propagating wave with time factor $e^{j \omega t}$ assumed. The Hankel function $H_{0}^{(2)}(k r)$ has a singularity at $r=0$, with asymptotic form shown in Eqs. (89)-(91) in Appendix B. In boundary element methods, the boundary is divided into discrete boundary elements. The distribution of $u$ and $(\nabla u) \cdot \boldsymbol{n}$ on 
each element is modeled by means of shape functions, according to a discretization scheme such as assignment to constant, linear, or higher order elements. By locating the field point $\boldsymbol{y}$ at every boundary node, we can obtain simultaneous equations, and determine the unknown quantities along the boundaries. When $\boldsymbol{y}$ approaches $\boldsymbol{x}$ in a boundary element, we should pay attention because both integrands $u^{*}$ and $\left(\nabla u^{*}\right) \cdot \boldsymbol{n}$ are singular. However, their integral does not diverge. In the case of $u^{*}$ the singularity is only on the order of $\log r$, and this integral becomes $r(\log r-1)$. Since it approaches 0 as $r$ approaches 0 , the singularity disappears. This kind of singularity is called a weakly singularity. In the case of the other integrand $\left(\nabla u^{*}\right) \cdot \boldsymbol{n}$, there is a stronger singularity. Introducing a unit vector between $\boldsymbol{x}$ and $\boldsymbol{y}$ as $\boldsymbol{e}_{r} \triangleq(\boldsymbol{x}-\boldsymbol{y}) / r$, we can rewrite the kernel as $\left(\nabla u^{*}\right) \cdot \boldsymbol{n}=\frac{\partial u^{*}}{\partial r} \boldsymbol{e}_{r} \cdot \boldsymbol{n}$. Since the vector $\boldsymbol{e}_{r}$ is perpendicular to the normal vector $\boldsymbol{n}$ near the singular point $\boldsymbol{y}$, the inner vector product, $\boldsymbol{e}_{r} \cdot \boldsymbol{n}$, becomes 0 ; therefore, the singularity also disappears. Thus, Eq. (3) does not necessitate inclusion of any singular integrals, and it requires only attention to ensure the accuracy of integration.

Next, let us consider the gradient of the wave field at the boundary nodes. Taking the gradient of Eq. (3) with respect to the field point $\boldsymbol{y}$, we can obtain the following equation,

$$
\begin{aligned}
\nabla_{y}[c(\boldsymbol{y}) u(\boldsymbol{y})]=\oint_{\Gamma} & {\left[\left(\nabla_{y} u^{*}(\boldsymbol{x} ; \boldsymbol{y})\right)(\nabla u(\boldsymbol{x})) \cdot \boldsymbol{n}\right.} \\
& \left.-u(\boldsymbol{x})\left(\nabla_{y} \nabla u^{*}(\boldsymbol{x} ; \boldsymbol{y})\right) \cdot \boldsymbol{n}\right] d \Gamma,
\end{aligned}
$$

where the $\nabla_{y}$ means the gradient with respect to $y$. The respective gradients of both fundamental solutions, $\nabla_{y} u^{*}$ and $\nabla_{y} \nabla u^{*}$, show stronger singularities than CBIE representation in Eq. (3). These singularities cannot be regularized simply because the aforementioned orthogonality of $\boldsymbol{n}$ and $\boldsymbol{e}_{r}$ does not apply to Eq. (6). This type of singularity is called a hyper-singularity, and the equation is called hypersingular boundary integral equation (HBIE). 


\section{Regularization of the hypersingular integral related to a gradient field}

\subsection{Hypersingular term}

Since the quantity $c(y)$ in Eq. (4) is not dependent on either the wave number $k$ or any field distributions, we can evaluate it by a Laplace equation, which is identical to the Eq. (1), in terms of $k=0$. Assuming the field is uniform, we can obtain $c(y)$ as the subsequent boundary integral representation [29], which is called the equi-potential condition:

$$
c(\boldsymbol{y})=-\oint_{\Gamma} \nabla u_{L}^{*}(\boldsymbol{x} ; \boldsymbol{y}) \cdot \boldsymbol{n} d \Gamma,
$$

where $u_{L}^{*}$ is a fundamental solution of the Laplace equation. Substituting this relation into Eq. (3), we can reduce to the following expression,

$$
\oint_{\Gamma}\left[q^{*}(\boldsymbol{x} ; \boldsymbol{y}) u(\boldsymbol{x})-q_{L}^{*}(\boldsymbol{x} ; \boldsymbol{y}) u(\boldsymbol{y})-u^{*}(\boldsymbol{x} ; \boldsymbol{y}) q(\boldsymbol{x})\right] d \Gamma=0,
$$

where $q, q^{*}$, and $q_{L}^{*}$ are the normal derivatives of $u, u^{*}$, and $u_{L}^{*}$, respectively. The last term of the integrand has only a weakly singularity as shown in the previous section. In contrast, both the first and the second term have stronger singularities. However, the singularity of sum of them is canceled as follows. Since, the fundamental solution of $u_{L}^{*}$ can be given as

$$
u_{L}^{*}=-\frac{1}{2 \pi} \log r
$$

the singularity of $u_{L}^{*}$ is same as $u^{*}$. Therefore, the difference between the fundamental solutions,

$$
\delta u^{*} \triangleq u^{*}-u_{L}^{*}
$$

has no singularity with $O(\log r)$ even if $\boldsymbol{y}=\boldsymbol{x}$, and it has the highest order term of $O(1)$. Thus, the normal derivative of $\delta u^{*}$ has no singularity, and Eq. (8) is a regular boundary integral equation. In fact, we can choose the $O(1)$ term of $\delta u^{*}$ so that it will also be canceled because the constant term in the fundamental solution can be chosen arbitrarily. In this case we can find that the significant order of $\delta u^{*}$ around $r \sim 0$ becomes 
$O\left(r^{2} \log r\right)$ from Eqs. (89)-(91) in Appendix B. The regularization scheme has been also used for HBIE regularization in past studies [2, 9-12, 16-18, 20].

Since Eq. (8) is satisfied for any boundary shapes, we can modify the boundary to exclude the point $\boldsymbol{y}$ as shown in Fig. 1. This modification is achieved as follows. First, the original boundary is divided into two types of boundary sections: the non-singular boundary section, $\Gamma_{n}$, and the singular one. Next, the singular boundary section including the singular point $\boldsymbol{y}$ is further separated into three sections to exclude $\boldsymbol{y}$ : the first is a part of circle section $\Gamma_{\varepsilon}$ with infinitesimal radius $\varepsilon$, the second is $\Gamma_{a}$ connecting from one of end points of $\Gamma_{\varepsilon}$ to the end point of $\Gamma_{n}$, and the last is $\Gamma_{b}$ connecting from the other end point of $\Gamma_{\varepsilon}$ to the other end point of $\Gamma_{n}$. The original boundary is redefined with limiting procedure as

$$
\Gamma=\Gamma_{n} \cup \lim _{\varepsilon \rightarrow 0}\left[\Gamma_{a} \cup \Gamma_{b} \cup \Gamma_{\varepsilon}\right] .
$$

Taking the gradient with respect to $y$ of Eq. (8), and considering the integral of $q_{L}^{*}$ disappears by Eq. (7) when the $\boldsymbol{y}$ is located outside the domain in the configuration, we can transform the boundary integral equation to

$$
\oint_{\Gamma}\left[\left(\nabla_{y} q^{*}\right) u-\left(\nabla_{y} q_{L}^{*}\right) u(\boldsymbol{y})-\left(\nabla_{y} u^{*}\right) q\right] d \Gamma=\mathbf{0}
$$

In the case where the gradient operator with respect to $y$ is applied to the functions that depend on only the distance $r$ such as $u^{*}$ or $u_{L}^{*}$, the result is represented by the gradient with respect to $\boldsymbol{x}$ with an opposite sign because the unit vectors of such functions have an opposite direction to each other.

$$
\begin{aligned}
& \nabla_{y} u^{*}=-\nabla u^{*}=-\frac{\partial u^{*}}{\partial r} \boldsymbol{e}_{r}, \\
& \nabla_{y} u_{L}^{*}=-\nabla u_{L}^{*}=-\frac{\partial u_{L}^{*}}{\partial r} \boldsymbol{e}_{r} .
\end{aligned}
$$

In contrast, the gradients of both $q^{*}$ and $q_{L}^{*}$ are not functions that depend only on the distance, $r$, because they also depend on the vector's inner product, $\boldsymbol{e}_{r} \cdot \boldsymbol{n}$. However, considering the relations of $\boldsymbol{e}_{r}=(\boldsymbol{x}-\boldsymbol{y}) / r$ and $\nabla_{y} \boldsymbol{y} \cdot \boldsymbol{n}=\boldsymbol{n}$, we can obtain the following expression,

$$
\nabla_{y} q^{*}=\left(-\frac{\partial^{2} u^{*}}{\partial r^{2}}+\frac{1}{r} \frac{\partial u^{*}}{\partial r}\right) \boldsymbol{e}_{r} \boldsymbol{e}_{r} \cdot \boldsymbol{n}-\frac{1}{r} \frac{\partial u^{*}}{\partial r} \boldsymbol{n}
$$


Since $u^{*}$ satisfies the following Helmholtz equation expressed in circular polar coordinates, the second order derivative of $u^{*}$ can be replaced by the first order derivative and the $u^{*}$ itself.

$$
\frac{\partial^{2} u^{*}}{\partial r^{2}}+\frac{1}{r} \frac{\partial u^{*}}{\partial r}+k^{2} u^{*}=0
$$

Thus, we can obtain $\nabla_{y} q^{*}$; furthermore, putting $k=0$ into the result of $\nabla_{y} q^{*}$, we can also obtain $\nabla_{y} q_{L}^{*}$ as follows:

$$
\begin{aligned}
& \nabla_{y} q^{*}=\left(k^{2} u^{*}+\frac{2}{r} \frac{\partial u^{*}}{\partial r}\right) \boldsymbol{e}_{r} \boldsymbol{e}_{r} \cdot \boldsymbol{n}-\frac{1}{r} \frac{\partial u^{*}}{\partial r} \boldsymbol{n}, \\
& \nabla_{y} q_{L}^{*}=\frac{2}{r} \frac{\partial u_{L}^{*}}{\partial r} \boldsymbol{e}_{r} \boldsymbol{e}_{r} \cdot \boldsymbol{n}-\frac{1}{r} \frac{\partial u_{L}^{*}}{\partial r} \boldsymbol{n} .
\end{aligned}
$$

The three integrals on the left-hand side in Eq. (12) can be written as follows.

$$
\begin{aligned}
\boldsymbol{I}_{1} & \triangleq \oint_{\Gamma}\left(\nabla_{y} q^{*}\right) u d \Gamma \\
& =\oint_{\Gamma}\left\{\left(k^{2} u^{*}+\frac{2}{r} \frac{\partial u^{*}}{\partial r}\right) \boldsymbol{e}_{r} \boldsymbol{e}_{r} \cdot \boldsymbol{n}-\frac{1}{r} \frac{\partial u^{*}}{\partial r} \boldsymbol{n}\right\} u d \Gamma, \\
\boldsymbol{I}_{2} & \triangleq-\oint_{\Gamma}\left(\nabla_{y} q_{L}^{*}\right) u(\boldsymbol{y}) d \Gamma \\
& =-u(\boldsymbol{y}) \oint_{\Gamma}\left\{\frac{2}{r} \frac{\partial u_{L}^{*}}{\partial r} \boldsymbol{e}_{r} \boldsymbol{e}_{r} \cdot \boldsymbol{n}-\frac{1}{r} \frac{\partial u_{L}^{*}}{\partial r} \boldsymbol{n}\right\} d \Gamma, \\
\boldsymbol{I}_{3} & \triangleq-\oint_{\Gamma}\left(\nabla_{y} u^{*}\right) q d \Gamma=\oint_{\Gamma} \frac{\partial u^{*}}{\partial r} \boldsymbol{e}_{r} q d \Gamma .
\end{aligned}
$$

The orders of integration kernels in both $\boldsymbol{I}_{1}$ and $\boldsymbol{I}_{2}$ are $O\left(r^{-2}\right)$ and that of $\boldsymbol{I}_{3}$ is $O\left(r^{-1}\right)$ for the vicinity around $r \sim 0$.

\subsection{Integral along the boundary $\Gamma_{a}$ and $\Gamma_{b}$}

We assume a linear shaped boundary element for $\Gamma_{a}$ and $\Gamma_{b}$ in Eqs. (19)-(21), with nodes located at both ends of each element. We also consider that the normal derivative $q=\nabla u \cdot \boldsymbol{n}$ varies linearly within the element, following a linear element discretization scheme, so that $q$ can be represented by the following Taylor expansion; similar formulations to regularization have been applied in [15-18, 20, 22, 23].

$$
q=\nabla u \cdot \boldsymbol{n} \sim(\nabla u \cdot \boldsymbol{n})_{\boldsymbol{y}}+r \boldsymbol{e}_{r} \cdot(\nabla \nabla u \cdot \boldsymbol{n})_{\boldsymbol{y}} .
$$


Since the order of $q$ is less than that of $u$, the distribution of $u$ should be expressed including the second order as follows, to ensure the accuracy of $q$ being equivalent to that of $u$.

$$
u \sim u(\boldsymbol{y})+r \boldsymbol{e}_{r} \cdot(\nabla u)_{y}+\frac{r^{2}}{2} \boldsymbol{e}_{r} \cdot \boldsymbol{e}_{r} \cdot(\nabla \nabla u)_{y}
$$

In the case where the integration point $\boldsymbol{x}$ is located on the linear shaped boundary $\Gamma_{a}$ or $\Gamma_{b}$, the unit vector $\boldsymbol{e}_{r}=(\boldsymbol{x}-\boldsymbol{y}) / r$ is identical to the unit tangential vector $\boldsymbol{\tau}_{\gamma}$, where the suffix $\gamma$ denotes either $a$ or $b$ (see Fig. 1). Since $\boldsymbol{\tau}_{\gamma}$ is perpendicular to $\boldsymbol{n}_{\gamma}$, the vector $\boldsymbol{e}_{r} \boldsymbol{e}_{r} \cdot \boldsymbol{n}$ that appeared at the first term on the right-hand side of Eqs. (19) and (20) vanishes.

The summation of the non-zero integration kernels of Eqs. (19)-(21) is evaluated using Eqs. (23), (22) and (10), as follows:

$$
\begin{aligned}
-\frac{1}{r} \frac{\partial u^{*}}{\partial r} u \boldsymbol{n}_{\gamma} & +\frac{1}{r} \frac{\partial u_{L}^{*}}{\partial r} u(\boldsymbol{y}) \boldsymbol{n}_{\gamma}+\frac{\partial u^{*}}{\partial r} q \boldsymbol{\tau}_{\gamma} \\
= & \frac{\partial u^{*}}{\partial r}\left(\boldsymbol{n}_{\gamma} \times \boldsymbol{\tau}_{\gamma}\right) \times(\nabla u)_{\boldsymbol{y}}-\frac{1}{r} \frac{\partial \delta u^{*}}{\partial r} u(\boldsymbol{y}) \boldsymbol{n}_{\gamma} \\
& +r \frac{\partial u^{*}}{\partial r}\left(-\left.\frac{1}{2} \boldsymbol{n}_{\gamma} \frac{\partial^{2} u}{\partial \tau_{\gamma}^{2}}\right|_{\boldsymbol{y}}+\left.\boldsymbol{\tau}_{\gamma} \frac{\partial^{2} u}{\partial \tau_{\gamma} \partial n_{\gamma}}\right|_{\boldsymbol{y}}\right),
\end{aligned}
$$

where the representation of the first term with vector triple product is derived from the formula $\left(\boldsymbol{n}_{\gamma} \times \boldsymbol{\tau}_{\gamma}\right) \times(\nabla u)_{\boldsymbol{y}}=\left(\boldsymbol{n}_{\gamma} \cdot(\nabla u)_{\boldsymbol{y}}\right) \boldsymbol{\tau}_{\gamma}-\left(\boldsymbol{\tau}_{\gamma} \cdot(\nabla u)_{\boldsymbol{y}}\right) \boldsymbol{n}_{\gamma}$. The order of the singularity of each term $\frac{\partial u^{*}}{\partial r}, \frac{1}{r} \frac{\partial \delta u^{*}}{\partial r}$, and $r \frac{\partial u^{*}}{\partial r}$ is $O\left(r^{-1}\right), O(\log r)$, and $O(1)$, respectively. Although the second term on the right-hand side becomes a weakly singular integrand, the first term is still singular.

This singularity of the first term, can be removed by considering the summation of the integrals of $\Gamma_{a}$ and $\Gamma_{b}$. The factor of the singular term contains the vector product of $\boldsymbol{n}_{\gamma} \times \boldsymbol{\tau}_{\gamma}$. They have mutually opposite signs, and their lengths are same;

$$
\boldsymbol{n}_{\gamma} \times \boldsymbol{\tau}_{\gamma}=\boldsymbol{e}_{3 \gamma}=\left\{\begin{array}{lll}
-\boldsymbol{e}_{3} & : & (\gamma=a) \\
+\boldsymbol{e}_{3} & : & (\gamma=b)
\end{array},\right.
$$

where $\boldsymbol{e}_{3}$ is one of the unit vectors in the Cartesian coordinate system that is perpendicular to the 2-dimensional domain under consideration, as shown in Fig. 1. 
Using this property, the singular integral is canceled and the result is easily evaluated:

$$
\begin{aligned}
\sum_{\gamma=a, b} & \int_{\Gamma_{\gamma}} \frac{\partial u^{*}}{\partial r}\left(\boldsymbol{n}_{\gamma} \times \boldsymbol{\tau}_{\gamma}\right) d \Gamma \times(\nabla u)_{\boldsymbol{y}} \\
& =\left[-u^{*}\left(L_{a}\right)+u^{*}\left(L_{b}\right)\right] \boldsymbol{e}_{3} \times(\nabla u)_{\boldsymbol{y}} \\
& =\left[-u^{*}\left(L_{a}\right)+u^{*}\left(L_{b}\right)\right]\left(-\boldsymbol{e}_{1} \boldsymbol{e}_{2}+\boldsymbol{e}_{2} \boldsymbol{e}_{1}\right) \cdot(\nabla u)_{\boldsymbol{y}},
\end{aligned}
$$

where $L_{a}$ and $L_{b}$ represent the size of elements $\Gamma_{a}$ and $\Gamma_{b}$, respectively. It should be noted that the computation of the sum of the integral along the singular elements $\Gamma_{a}$ and $\Gamma_{b}$ is obtained solely by evaluations of $u^{*}$ at one end of each element, and it requires no numerical integrations. Moreover, limitations regarding element size or smoothness are not imposed in this formulation; it is applicable for different sizes of boundary elements, and also applicable for corners. To simplify notations in later discussion we introduce an abbreviation of a difference operator between a function $f$ related to $\Gamma_{a}$ and that related to $\Gamma_{b}$, as $\underset{\gamma: a-b}{\operatorname{Diff}}\left[f_{\gamma}\right] \triangleq f_{a}-f_{b}$. The coefficients of $(\nabla u)_{y}$ on the left-hand side of Eq. (26) are rewritten as the following dyadic tensor,

$$
\begin{aligned}
\overleftrightarrow{\mathbf{C}_{a \cup b}}(\boldsymbol{y}) & \triangleq-\underset{\gamma: a-b}{\operatorname{Diff}}\left[u^{*}\left(L_{\gamma}\right)\right]\left(-\boldsymbol{e}_{1} \boldsymbol{e}_{2}+\boldsymbol{e}_{2} \boldsymbol{e}_{1}\right) \\
& =\left(\begin{array}{cc}
0 & \underset{\gamma: a-b}{\operatorname{Diff}}\left[u^{*}\left(L_{\gamma}\right)\right] \\
-\operatorname{Diff}_{\gamma: a-b}\left[u^{*}\left(L_{\gamma}\right)\right] & 0
\end{array}\right) .
\end{aligned}
$$

Next, let us consider the second and third terms on the right-hand side of Eq. (24). The second order derivatives at the third term can be expressed as a linear combination of $u$ and $q$ at associated points, which are the singular node $\boldsymbol{y}$ and its adjacent nodes $\boldsymbol{x}_{\gamma}$, as shown in Eq. (81) and Eqs. (83)-(85) in Appendix A. Also the second term is expressed by $u(y)$ itself. Consequently, the integrals of terms of Eq. (24) are expressed by $u$ and $q$ but not $\nabla u$, so we introduce the following vector definition.

$$
\begin{aligned}
\boldsymbol{J}_{\gamma}(u, q) \triangleq-\int_{0}^{L_{\gamma}} \frac{1}{r} \frac{\partial \delta u^{*}}{\partial r} d r u(\boldsymbol{y}) \boldsymbol{n}_{\gamma} \\
\quad+\int_{0}^{L_{\gamma}} r \frac{\partial u^{*}}{\partial r} d r\left(-\left.\frac{1}{2} \boldsymbol{n}_{\gamma} \frac{\partial^{2} u}{\partial \tau_{\gamma}^{2}}\right|_{\boldsymbol{y}}+\left.\boldsymbol{\tau}_{\gamma} \frac{\partial^{2} u}{\partial \tau_{\gamma} \partial n_{\gamma}}\right|_{\boldsymbol{y}}\right) .
\end{aligned}
$$

For the sake of accurate evaluations of both the weakly singular integral and the regular integral, we can apply the analytical integral shown in Appendix B. 
Thus, the summation of the entire integral along $\Gamma_{a}$ and $\Gamma_{b}$ is represented as

$$
\boldsymbol{I}_{a \cup b} \triangleq \sum_{\gamma=a, b} \sum_{i=1}^{3} \boldsymbol{I}_{i, \gamma}=\overleftrightarrow{\mathbf{C}_{a \cup b}}(\boldsymbol{y}) \cdot(\nabla u)_{\boldsymbol{y}}+\sum_{\gamma=a, b} \boldsymbol{J}_{\gamma}(u, q)
$$

\subsection{Integral along the boundary $\Gamma_{\varepsilon}$}

The integration path $\Gamma_{\varepsilon}$ can be given by $\varepsilon d \theta$ where $\varepsilon$ is an infinitesimal constant, and $\theta$ is the azimuthal angle from the direction $\boldsymbol{e}_{1}$ in the Cartesian coordinate system. The angle $\theta$ varies from $\theta_{b}$ to $\theta_{a}$ with the interior angle $\Delta \theta=\theta_{a}-\theta_{b}>0$. Since $\boldsymbol{e}_{r}=-\boldsymbol{n}_{\varepsilon}$ is satisfied throughout the path, each of the integrals (Eqs. (19)-(21)) along $\Gamma_{\varepsilon}$ is readily evaluated by applying a similar procedure to that used in the reduction of Eq. (24). The terms with non-zero values in the integrals are as follows:

$$
\begin{aligned}
& \left.\boldsymbol{I}_{1, \varepsilon} \triangleq \lim _{\varepsilon \rightarrow 0} \int_{\theta_{b}}^{\theta_{a}} \frac{\partial u^{*}}{\partial r}\right|_{\varepsilon} \boldsymbol{n}_{\varepsilon}\left(u(\boldsymbol{y})-\varepsilon \boldsymbol{n}_{\varepsilon} \cdot(\nabla u)_{\boldsymbol{y}}\right) d \theta, \\
& \boldsymbol{I}_{2, \varepsilon} \triangleq-\left.u(\boldsymbol{y}) \lim _{\varepsilon \rightarrow 0} \int_{\theta_{b}}^{\theta_{a}} \frac{\partial u_{L}^{*}}{\partial r}\right|_{\varepsilon} \boldsymbol{n}_{\varepsilon} d \theta, \\
& \boldsymbol{I}_{3, \varepsilon} \triangleq-\lim _{\varepsilon \rightarrow 0} \int_{\theta_{b}}^{\theta_{a}}\left(\left.\frac{\partial u^{*}}{\partial r}\right|_{\varepsilon} \boldsymbol{n}_{\varepsilon} \boldsymbol{n}_{\varepsilon} \cdot(\nabla u)_{\boldsymbol{y}} \varepsilon\right) d \theta .
\end{aligned}
$$

Since the singularity of $\frac{\partial \delta u^{*}}{\partial r}$ is $O(r \log r)$, the coefficient of $u(y)$ vanishes with $\varepsilon \rightarrow 0$; therefore, the summation of these integrals are simplified as

$$
\boldsymbol{I}_{\varepsilon} \triangleq \sum_{i=1}^{3} \boldsymbol{I}_{i, \varepsilon}=-\lim _{\varepsilon \rightarrow 0}\left(\left.\varepsilon \frac{\partial u^{*}}{\partial r}\right|_{\varepsilon}\right) \int_{\theta_{b}}^{\theta_{a}} 2 \boldsymbol{n}_{\varepsilon} \boldsymbol{n}_{\varepsilon} d \theta \cdot(\nabla u)_{\boldsymbol{y}}
$$

Furthermore, $\boldsymbol{I}_{\varepsilon}$ is a regularized term because the singularity of $\frac{\partial u^{*}}{\partial r}$ is $O\left(r^{-1}\right)$, and the coefficient of the integral can be evaluated as

$$
\lim _{\varepsilon \rightarrow 0}\left(\left.\varepsilon \frac{\partial u^{*}}{\partial r}\right|_{\varepsilon}\right)=\frac{-1}{2 \pi}
$$

The vector $\boldsymbol{n}_{\varepsilon}$ and the dyad $\boldsymbol{n}_{\varepsilon} \boldsymbol{n}_{\varepsilon}$ are written in terms of unit vectors in the Cartesian coordinate system, $\boldsymbol{e}_{1}$ and $\boldsymbol{e}_{2}$, as follows:

$$
\begin{aligned}
& \boldsymbol{n}_{\varepsilon}=-\boldsymbol{e}_{r}=-\left(\cos \theta \boldsymbol{e}_{1}+\sin \theta \boldsymbol{e}_{2}\right) \\
& \boldsymbol{n}_{\varepsilon} \boldsymbol{n}_{\varepsilon}=\cos ^{2} \theta \boldsymbol{e}_{1} \boldsymbol{e}_{1}+\cos \theta \sin \theta\left(\boldsymbol{e}_{1} \boldsymbol{e}_{2}+\boldsymbol{e}_{2} \boldsymbol{e}_{1}\right)+\sin ^{2} \theta \boldsymbol{e}_{2} \boldsymbol{e}_{2}
\end{aligned}
$$


Evaluating the definite integral with respect to $\theta$ using these equations, we can obtain the following result:

$$
\begin{aligned}
\boldsymbol{I}_{\varepsilon}= & \underset{\gamma: a-b}{\operatorname{Diff}}\left[\frac{\theta_{\gamma}}{2 \pi}\right] \overleftrightarrow{\mathbf{1}}+\underset{\gamma: a-b}{\operatorname{Diff}}\left[\frac{\sin 2 \theta_{\gamma}}{4 \pi}\right]\left(\boldsymbol{e}_{1} \boldsymbol{e}_{1}-\boldsymbol{e}_{2} \boldsymbol{e}_{2}\right) \\
& \left.+\underset{\gamma: a-b}{\operatorname{Diff}}\left[\frac{-\cos 2 \theta_{\gamma}}{4 \pi}\right]\left(\boldsymbol{e}_{1} \boldsymbol{e}_{2}+\boldsymbol{e}_{2} \boldsymbol{e}_{1}\right)\right) \cdot(\nabla u)_{\boldsymbol{y}} \\
\triangleq & \overleftrightarrow{\mathbf{C}_{\varepsilon}}(\boldsymbol{y}) \cdot(\nabla u)_{\boldsymbol{y}},
\end{aligned}
$$

where $\overleftrightarrow{\mathbf{1}}$ denotes an identical dyadic tensor. The coefficient of $\overleftrightarrow{\mathbf{1}}$ is equal to that of CBIE. The coefficient dyad $\overleftrightarrow{\mathbf{C}_{\varepsilon}}(\boldsymbol{y})$ is same as that for Laplace equation [22, 23] (note these references contain a few mistakes in representations of the coefficients).

Furthermore, Chen et al. [24] shows similar coefficients called free term, but their representation is given by two normal derivatives with different normal directions at a corner.

\subsection{Regularized boundary integral equation for gradient field}

Using Eqs. (29) and (37), the integral equation of the gradient given in Eq. (12) is expressed by only the regularized terms as follows:

$$
\overleftrightarrow{\mathbf{C}}(\boldsymbol{y}) \cdot \nabla u(\boldsymbol{y})=-\boldsymbol{J}(u, q)
$$

The right-hand side, $\boldsymbol{J}(u, q)$, is composed of $\boldsymbol{J}_{\gamma}(u, q)$ and $\boldsymbol{J}_{n}(u, q)$, where $\boldsymbol{J}_{\gamma}(u, q)$ is the regularized result of the hypersingular integral shown in Eq. (28) that is not dependent on $\nabla u$, and $\boldsymbol{J}_{n}(u, q)$ is the integral along the non-singular boundary elements. The coefficient dyad $\overleftrightarrow{\mathbf{C}}$ is the regularized result of hypersingular integrals associated with $\nabla u$.

$$
\begin{aligned}
& \boldsymbol{J}(u, q) \triangleq \sum_{\gamma=a, b} \boldsymbol{J}_{\gamma}(u, q)+\boldsymbol{J}_{n}(u, q), \\
& \boldsymbol{J}_{n}(u, q) \triangleq \int_{\Gamma_{n}}\left\{\left(\nabla_{y} q^{*}\right) u-\left(\nabla_{y} q_{L}^{*}\right) u(\boldsymbol{y})-\left(\nabla_{y} u^{*}\right) q\right\} d \Gamma, \\
& \overleftrightarrow{\mathbf{C}}(\boldsymbol{y}) \triangleq \overleftrightarrow{\mathbf{C}_{\varepsilon}}(\boldsymbol{y})+\overleftrightarrow{\mathbf{C}_{a \cup b}}(\boldsymbol{y}) \\
& =\left(\begin{array}{c}
\operatorname{Diff}_{\gamma: a-b}\left[\frac{2 \theta_{\gamma}+\sin 2 \theta_{\gamma}}{4 \pi}\right] \\
\underset{\gamma: a-b}{\operatorname{Diff}}\left[\frac{-\cos 2 \theta_{\gamma}}{4 \pi}+u^{*}\left(L_{\gamma}\right)\right] \\
\underset{\gamma: a-b}{\operatorname{Diff}}\left[\frac{-\cos 2 \theta_{\gamma}}{4 \pi}-u^{*}\left(L_{\gamma}\right)\right] \underset{\gamma: a-b}{\operatorname{Diff}}\left[\frac{2 \theta_{\gamma}-\sin 2 \theta_{\gamma}}{4 \pi}\right]
\end{array}\right) .
\end{aligned}
$$


When we can assume that the element size $L_{\gamma}$ is small enough compared to the wavelength, the $\overleftrightarrow{\mathbf{C}}(\boldsymbol{y})$ is a regular matrix as shown in Appendix C. Therefore, the inverse matrix $\overleftrightarrow{\mathbf{C}^{-1}}(\boldsymbol{y})$ can be defined as Eq. (98). Operating the inverse matrix to Eq. (38), the following equation is derived,

$$
\nabla u(\boldsymbol{y})=-\overleftrightarrow{\mathbf{C}^{-1}}(\boldsymbol{y}) \cdot \boldsymbol{J}(u, q)
$$

Since the $\overleftrightarrow{\mathbf{C}^{-1}}(\boldsymbol{y})$ depends on only the boundary shape and the element size, once the quantities $u$ and $q$ are known, the gradient can be computed by an evaluation of the right-hand side as an explicit form.

In general problems either one of $u$ or $q$ on the boundary is given, but not the other. In this case, taking the scalar product of $\boldsymbol{n}_{\gamma}(\boldsymbol{y})$ and Eq. (42), we can obtain a Fredholm equation of the second type with respect to $q$ :

$$
q_{\gamma}(\boldsymbol{y})=-\boldsymbol{n}_{\gamma}(\boldsymbol{y}) \cdot \overleftrightarrow{\mathbf{C}^{-1}}(\boldsymbol{y}) \cdot \boldsymbol{J}(u, q)
$$

Similarly to CBIE, this equation can be solved after constructing a set of equations obtained by taking $\boldsymbol{y}$ for every boundary node.

Two kinds of error arise in solving such a set derived from Eq. (43): one is the error from the regularization of the hypersingular integral equation, and the other is the rounding error in solving simultaneous equations. In contrast, Eq. (42) only includes error from the regularization. Since another aim of this paper is estimation of the error due to the regularization, we will consider the evaluation of Eq. (42) in later sections.

\section{Error Estimation}

In the section 3 in order to derive the regularized equation of gradient field, we considered the second order derivatives of $u$ around the singular point in Eq. (23), and higher order derivatives were truncated. The error by interpolation using shape functions in the singular element, which is the error of Eq. (28), results from these truncated terms. In the integral of non-singular elements defined in Eq. (40) this error is also included. In this section we will roughly estimate these errors. We classify the error source terms as 
follows,

$$
\begin{aligned}
& \boldsymbol{J}_{\gamma}\left\{\partial_{\tau \tau}^{2} u\right\} \triangleq-\left.\frac{1}{2} \int_{0}^{L_{\gamma}} r \frac{\partial u^{*}}{\partial r} d r \frac{\partial^{2} u}{\partial \tau_{\gamma}^{2}}\right|_{\boldsymbol{y}} \boldsymbol{n}_{\gamma}, \\
& \left.\boldsymbol{J}_{\gamma}\left\{\partial_{\tau n}^{2} u\right\} \triangleq \int_{0}^{L_{\gamma}} r \frac{\partial u^{*}}{\partial r} d r \frac{\partial^{2} u}{\partial \tau_{\gamma} \partial n_{\gamma}}\right|_{y} \boldsymbol{\tau}_{\gamma} \\
& \boldsymbol{J}_{n_{i}}\{u\} \triangleq \int_{\Gamma_{n_{i}}}\left(\nabla_{y} q^{*}\right) u d \Gamma \\
& \boldsymbol{J}_{n_{i}}\{q\} \triangleq \int_{\Gamma_{n_{i}}}-\left(\nabla_{y} u^{*}\right) q d \Gamma
\end{aligned}
$$

where the identifiers $\gamma$ and $n_{i}$ show, the singular element $(\gamma \in\{a, b\})$ and the $i$-th non-singular boundary element, respectively, moreover, the argument in the bracket on the left-hand side of each equation shows the cause of error, in which $\partial_{\tau}$ and $\partial_{n}$ are abbreviation of derivative with respect to $\tau$ and $n$, respectively.

In order to facilitate estimation of the error we assume that $u$ is a plane wave, i.e., a D'Alembert's solution, which satisfies the following equations,

$$
\nabla u+j \boldsymbol{k} u=\mathbf{0} .
$$

The amplitude of this solution can be estimated as follows:

$$
|\nabla u|=k|u|, \quad\left|\partial_{n} u\right|=k|u||\cos \phi|, \quad\left|\partial_{\tau} u\right|=k|u||\sin \phi|,
$$

where $\phi$ is the angle between the wave vector $\boldsymbol{k}$ and the normal unit vector $\boldsymbol{n}$. The higher order derivatives satisfy similar relations.

Although the discussions in this section are only focused on a plane wave, it should be noted that the application can be expanded to the case where the field $u$ is expressed as a sum of plane waves. By considering a plane wave $u_{m}$ with wave vector $\boldsymbol{k}_{\boldsymbol{m}}$ that has the same magnitude as other plane waves but different directions, the amplitude of a gradient of the total field can be estimated as the following expanded relation:

$$
\begin{gathered}
u=\sum_{m=1}^{M} u_{m}, \quad \nabla u_{m}=-j \boldsymbol{k}_{\boldsymbol{m}} u_{m}, \quad\left|\boldsymbol{k}_{\boldsymbol{m}}\right|=k, \\
|\nabla u|=\left|\sum_{m=1}^{M} \boldsymbol{k}_{\boldsymbol{m}} u_{m}\right| \leq \sum_{m=1}^{M}\left|\boldsymbol{k}_{\boldsymbol{m}}\right| \cdot\left|u_{m}\right|=k \sum_{m=1}^{M}\left|u_{m}\right| \\
\leq M \max _{m}\left|u_{m}\right| .
\end{gathered}
$$




\subsection{Error due to integral including singular point}

Both the errors associated with Eqs. (44) and (45) are mainly affected by the errors of the second order derivatives with the discretization procedure because the integral can be evaluated with good accuracy by analytical integral expressed as Eq. (88) in Appendix B. The errors of the second order derivatives of which details are shown in Appendix D, depend on the geometries: sizes of two singular elements that both include the singular point $\boldsymbol{y}$, and the interior angle $\Delta \theta$ at $\boldsymbol{y}$. In subsequent discussion, the sizes of the singular elements are denoted using a parameter $\alpha$ as $L_{\gamma}=L$ and $L_{\gamma^{\prime}}=\alpha L$, where the subscript $\gamma$ is either one of $a$ or $b$, and $\gamma^{\prime}$ is the other one. In terms of the internal angle we consider two typical cases; a flat boundary $(\Delta \theta=\pi)$, and a corner with right angle $(\Delta \theta=\pi / 2)$. The followings are the estimated error of $\boldsymbol{J}_{\gamma}\left\{\partial_{\tau \tau}^{2} u\right\}$ and $\boldsymbol{J}_{\gamma}\left\{\partial_{\tau n}^{2} u\right\}$.

$$
\begin{aligned}
& \left|\Delta \boldsymbol{J}_{\gamma}\left\{\partial_{\tau \tau}^{2} u\right\}\right| \\
& \sim \begin{cases}\frac{(k L)^{2}|1-\alpha|}{12 \pi}\left|\sin ^{3} \phi\right||k u(\boldsymbol{y})| & \left(\begin{array}{l}
\Delta \theta=\pi \text { and } \\
|1-\alpha| \ll k L
\end{array}\right), \\
\frac{(k L)^{3}\left(1+\alpha^{3}\right)}{48 \pi(1+\alpha)}\left|\sin ^{4} \phi\right||k u(y)| & \left(\begin{array}{l}
\Delta \theta=\pi \text { and } \\
|1-\alpha| \ll k L
\end{array}\right) .\end{cases} \\
& \left|\Delta \boldsymbol{J}_{\gamma}\left\{\partial_{\tau \tau}^{2} u\right\}\right|=\frac{\alpha(k L)^{2}}{12 \pi}\left|\cos ^{3} \phi\right||k u(\boldsymbol{y})| \quad(\Delta \theta=\pi / 2), \\
& \left|\Delta \boldsymbol{J}_{\gamma}\left\{\partial_{\tau n}^{2} u\right\}\right|=\frac{(k L)^{2}}{4 \pi}\left|\sin ^{2} \phi \cos \phi\right||k u(\boldsymbol{y})|\left(\begin{array}{c}
\Delta \theta=\pi \text { or } \\
\Delta \theta=\pi / 2
\end{array}\right) .
\end{aligned}
$$

In the case of $L_{\gamma}=L_{\gamma^{\prime}}$, by comparing Eq. (51) and Eq. (53), we can find that the error due to $\left(\partial_{\tau n}^{2} u\right)_{y}$ is larger than the error due to $\left(\partial_{\tau \tau}^{2} u\right)_{y}$. Otherwise, the orders of the errors are same, $(k L)^{2}$.

The error $\left|\Delta \boldsymbol{J}_{\gamma}\left\{\partial_{\tau \tau}^{2} u\right\}\right|$ for the corner shown in Eq. (52) error decreases by taking smaller $\alpha$ at a glance. However, in the situation of exchanging $\gamma$ and $\gamma^{\prime}$, the error increases because the factor $\alpha$ is replaced by its reciprocal. Since the total integral given in Eq. (39) includes a sum of the case of $\left(\gamma, \gamma^{\prime}\right)=(a, b)$ and an exchanged case $\left(\gamma, \gamma^{\prime}\right)=(b, a)$, the factor of the error should be estimated as $\alpha+1 / \alpha$. Thus, when we 
choose smaller $\alpha$, the error of the total integral will not become smaller. Furthermore, in the case of $\alpha \sim 1$ the error for $\Delta \theta=\pi / 2$ (Eq. (52)) is larger than that for $\Delta \theta=\pi$ (Eq. (51)).

\subsection{Error due to the non-singular integral}

The error due to discretization in integral of non-singular elements appears as well as the integral of singular elements. Substituting the quantities $\nabla_{y} q^{*}(\boldsymbol{x} ; \boldsymbol{y})$ and $-\nabla_{y} u^{*}(\boldsymbol{x} ; \boldsymbol{y})$ to $\boldsymbol{\Psi}(\boldsymbol{x} ; \boldsymbol{y})$, and substituting $u(x)$ and $q(x)$ to $f(x)$, we can reduce to the general form of the non-singular integral in Eqs. (46) and (47) as follows:

$$
\boldsymbol{J}_{n_{i}}\{f\} \triangleq \int_{\boldsymbol{x}_{i}^{\prime}}^{\boldsymbol{x}_{i}^{\prime \prime}} \boldsymbol{\Psi}(\boldsymbol{x} ; \boldsymbol{y}) f(\boldsymbol{x}) d \Gamma,
$$

where $\boldsymbol{x}_{i}^{\prime}, \boldsymbol{x}_{i}^{\prime \prime}$ are both ends of the non-singular element $\Gamma_{n_{i}}$ and $\boldsymbol{y} \notin \Gamma_{n_{i}}$. The function $f$ can be expanded by a shape function $\zeta_{j}(\boldsymbol{x})$ as follows:

$$
f(\boldsymbol{x})=\sum_{j} f_{j} \zeta_{j}(\boldsymbol{x})+\Delta f(\boldsymbol{x}),
$$

where $\Delta f(\boldsymbol{x})$ means truncated terms in the discretizing procedure. When we can calculate the first term with good accuracy, the error due to the non-singular integral is determined by $\Delta f(\boldsymbol{x})$ at the second term;

$$
\Delta \boldsymbol{J}_{n_{i}}\{f\} \triangleq \int_{\boldsymbol{x}_{i}^{\prime}}^{\boldsymbol{x}_{i}^{\prime \prime}} \boldsymbol{\Psi}(\boldsymbol{x} ; \boldsymbol{y}) \Delta f(\boldsymbol{x}) d \Gamma .
$$

In the case of linear element, the significant term of $\Delta f(\boldsymbol{x})$ within truncated terms is the second order derivative of $f$,

$$
\Delta f(x)=\Delta f^{(2)}(x)=\frac{1}{2}\left(x-x_{i}\right) \cdot\left(x-x_{i}\right) \cdot(\nabla \nabla f)_{x_{i}},
$$

where $\boldsymbol{x}_{i}=\frac{1}{2}\left(\boldsymbol{x}_{i}^{\prime}+\boldsymbol{x}_{i}^{\prime \prime}\right)$. Since $u$ is assumed as a D'Alembert's solution shown in Eq. (48), the gradient of $f$, where $f$ is either $u$ or $q=\nabla u \cdot \boldsymbol{n}$, satisfies $\nabla f=-j \boldsymbol{k} f$. Moreover, putting $\boldsymbol{x}-\boldsymbol{x}_{i}=l \boldsymbol{\tau}_{i}$, we can obtain the error as,

$$
\Delta \boldsymbol{J}_{n_{i}}\{f\}=\frac{-\left(\boldsymbol{k} \cdot \boldsymbol{\tau}_{i}\right)^{2} f\left(\boldsymbol{x}_{i}\right)}{2} \int_{-L / 2}^{+L / 2} \boldsymbol{\Psi}\left(\boldsymbol{x}_{i}+l \boldsymbol{\tau}_{i} ; \boldsymbol{y}\right) l^{2} d l .
$$

In the case where $l \ll r_{i}\left(r_{i}\right.$ shows distance between $\boldsymbol{x}_{i}$ and $\left.\boldsymbol{y}\right)$, the $\boldsymbol{\Psi}$ can be considered as a constant vector,

$$
\Delta \boldsymbol{J}_{n_{i}}\{f\} \sim-\frac{L^{3}}{24}\left(\boldsymbol{k} \cdot \boldsymbol{\tau}_{i}\right)^{2} f\left(\boldsymbol{x}_{i}\right) \boldsymbol{\Psi}\left(\boldsymbol{x}_{i} ; \boldsymbol{y}\right)
$$


Since $\boldsymbol{\Psi}$ increases with decreasing $r_{i}$, the error from the non-singular element near the singular point $\boldsymbol{y}$ becomes significant. When we consider the case where $r_{i} \ll 1 / k$, the fundamental solution $\Psi$, i.e., $\nabla_{y} u^{*}$ or $\nabla_{y} q^{*}$ given by Eqs. (13) and (17) are reduced approximately as follows:

$$
\begin{aligned}
\nabla_{y} u^{*} & =-\boldsymbol{e}_{r} \frac{\partial u^{*}}{\partial r} \sim \boldsymbol{e}_{\boldsymbol{r}_{i}} \frac{1}{2 \pi r_{i}}, \\
\nabla_{y} q^{*} & =\left(k^{2} u^{*}+\frac{2}{r} \frac{\partial u^{*}}{\partial r}\right)\left(\boldsymbol{n}_{i} \cdot \boldsymbol{e}_{r}\right) \boldsymbol{e}_{r}-\frac{1}{r} \frac{\partial u^{*}}{\partial r} \boldsymbol{n}_{i} \\
& \sim-\frac{2 \boldsymbol{e}_{\boldsymbol{r}_{i}}\left(\boldsymbol{n}_{i} \cdot \boldsymbol{e}_{\boldsymbol{r}_{i}}\right)-\boldsymbol{n}_{i}}{2 \pi r_{i}^{2}}=-\frac{\boldsymbol{n}_{i} \cos 2 \psi_{i}+\boldsymbol{\tau}_{i} \sin 2 \psi_{i}}{2 \pi r_{i}^{2}}
\end{aligned}
$$

where $\cos \psi_{i}=\boldsymbol{n}_{i} \cdot \boldsymbol{e}_{\boldsymbol{r}_{i}}, \sin \psi_{i}=\boldsymbol{\tau}_{i} \cdot \boldsymbol{e}_{\boldsymbol{r}_{i}}$. Introducing $\beta$ as $\beta=r_{i} / L$ and substituting the above equations and Eq. (49) into Eq. (59), we can finally estimate the error due to the non-singular integral as follows:

$$
\begin{aligned}
\left|\Delta \boldsymbol{J}_{n_{i}}\{q\}\right| \sim \frac{(k L)^{2}\left|\sin ^{2} \phi \cos \phi\right|}{48 \beta \pi}\left|k u\left(\boldsymbol{x}_{i}\right)\right|, \\
\left|\Delta \boldsymbol{J}_{n_{i}}\{u\}\right| \sim \frac{k L\left|\sin ^{2} \phi\right|}{48 \beta^{2} \pi}\left|k u\left(\boldsymbol{x}_{i}\right)\right| .
\end{aligned}
$$

These errors become larger for smaller $\beta$. Since $\left|\Delta \boldsymbol{J}_{n_{i}}\{u\}\right|$ is only proportional to the first order of $k L$, it becomes larger than the error due to the singular integral shown in the previous subsection.

\subsection{Total error of the gradient field}

In the above subsections we have shown the estimation of the error due to the discretization for components of $\boldsymbol{J}$. The total error of the gradient field $\nabla u$ is estimated by operating $\overleftrightarrow{\mathbf{C}^{-1}}$ on the sum of the errors of the components:

$$
\begin{aligned}
\Delta\{\nabla u\} \triangleq-\overleftrightarrow{\mathbf{C}^{-1}} \cdot \Delta \boldsymbol{J}, & \\
\Delta \boldsymbol{J} \triangleq \sum_{\gamma=a, b}\left[\Delta \boldsymbol{J}_{\gamma}\left\{\partial_{\tau \tau}^{2} u\right\}\right. & \left.+\Delta \boldsymbol{J}_{\gamma}\left\{\partial_{\tau n}^{2} u\right\}\right] \\
& +\sum_{i}\left[\Delta \boldsymbol{J}_{n_{i}}\{u\}+\Delta \boldsymbol{J}_{n_{i}}\{q\}\right] .
\end{aligned}
$$

Since the component of $\Delta \boldsymbol{J}$ is generally unknown, we apply the norm of the inverse 
matrix to estimate the total error.

$$
\begin{aligned}
& |\Delta\{\nabla u(\boldsymbol{y})\}|=\left|\overleftrightarrow{\mathbf{C}^{-1}} \Delta \boldsymbol{J}\right| \leq\left\|\overleftrightarrow{\mathbf{C}^{-1}}\right\||\Delta \boldsymbol{J}|, \\
& \left\|\overleftrightarrow{\mathbf{C}^{-1}}\right\|=\frac{1}{|\operatorname{det} \overleftrightarrow{\mathbf{C}}|}\left(\left|\frac{\Delta \theta}{2 \pi}\right|+\left|\operatorname{Diff}_{\gamma: a-b}\left[u^{*}\left(L_{\gamma}\right)\right]\right|+\left|\frac{\sin \Delta \theta}{2 \pi}\right|\right),
\end{aligned}
$$

where the estimation of the norm $\left\|\overleftrightarrow{\mathbf{C}^{-1}}\right\|$ is shown in Appendix C. The total error is magnified by this norm. Results of some typical cases are shown in bellow.

$$
\begin{aligned}
& (\Delta \theta=\pi) \quad\left\|\overleftrightarrow{\mathbf{C}^{-1}}\right\| \leq \frac{2\left(1+\left|\underset{\gamma: a-b}{\operatorname{Diff}}\left[u^{*}\left(L_{\gamma}\right)\right]\right|\right)}{\left|1+\left(\underset{\gamma: a-b}{2 \operatorname{Diff}}\left[u^{*}\left(L_{\gamma}\right)\right]\right)^{2}\right|} \\
& \left(\begin{array}{l}
\Delta \theta=\pi \\
L_{a}=L_{b}
\end{array}\right) \quad\left\|\overleftrightarrow{\mathbf{C}^{-1}}\right\|=2, \\
& \left(\Delta \theta=\frac{\pi}{2}\right) \quad\left\|\overleftrightarrow{\mathbf{C}^{-1}}\right\| \leq \frac{4 \pi\left(\pi+2+4 \pi\left|\operatorname{Diff}_{\gamma: a-b}\left[u^{*}\left(L_{\gamma}\right)\right]\right|\right)}{\left|\pi^{2}-4+16 \pi^{2}\left(\operatorname{Diff}_{\gamma: a-b}\left[u^{*}\left(L_{\gamma}\right)\right]\right)^{2}\right|}, \\
& \left(\begin{array}{c}
\Delta \theta=\frac{\pi}{2} \\
L_{a}=L_{b}
\end{array}\right) \quad\left\|\overleftrightarrow{\mathbf{C}^{-1}}\right\| \leq \frac{4 \pi}{\pi-2}
\end{aligned}
$$

In the case of $L_{a}=L_{b}$, we can find that the norm for $\Delta \theta=\pi$ is 2, while the maximum of the norm for $\Delta \theta=\pi / 2$ is almost 11 . This result suggests that the error for a corner with right angle in the worst case is magnified about 5 times larger than that for smooth boundary.

\section{Numerical Result}

In order to show the error of hypersingular integral in the proposed regularization, let us consider the case where true solution is given in whole domain. We adopt the following plane wave without scattering as the true solution $\widetilde{u}$ to compare the computational error of the hypersingular integral equation with the rough estimation 
discussed in the section 4;

$$
\widetilde{u}(\boldsymbol{x})=u_{0} e^{-j k \cdot \boldsymbol{x}},
$$

where $u_{0}$ is complex amplitude and $\boldsymbol{k}$ is a real wave vector. The true boundary values at boundary nodes $\boldsymbol{x}_{i}$ consisting of the field $\widetilde{u}\left(\boldsymbol{x}_{i}\right)$ and its normal derivatives $\widetilde{q}\left(\boldsymbol{x}_{i}\right)$ can be readily obtained. Substituting these boundary values to $u\left(\boldsymbol{x}_{i}\right)$ and $q\left(\boldsymbol{x}_{i}\right)$ on the right-hand side of Eq. (42), we can evaluate the gradient at the boundary nodes, $\nabla u\left(\boldsymbol{x}_{i}\right)$, as an explicit form. The reference of gradient at the boundary nodes is derived directly from the true field in Eq. (69) as $\nabla \widetilde{u}\left(\boldsymbol{x}_{i}\right)=-j \boldsymbol{k} \widetilde{u}\left(\boldsymbol{x}_{i}\right)$. The error of the gradient is defined as the difference of these gradients.

Since both of the results, $\nabla u$ by numerical integral and $\nabla \widetilde{u}$ by the reference, are dependent on position, it is difficult to fully capture the error. However, by normalizing the gradient with respect to the field itself, the true gradients can be converted to a position independent quantity. Similarly, the numerical gradient is normalized by the field as follows.

$$
\widetilde{\boldsymbol{e}_{k}} \triangleq \frac{\nabla \widetilde{u}}{-j|\boldsymbol{k}| \widetilde{u}}=\frac{\boldsymbol{k}}{|\boldsymbol{k}|}, \quad \boldsymbol{e}_{k} \triangleq \frac{\nabla u}{-j|\boldsymbol{k}| \widetilde{u}}
$$

The results shown below are represented in terms of these normalized differences,

$$
\Delta \boldsymbol{e}_{k} \triangleq \boldsymbol{e}_{k}-\widetilde{\boldsymbol{e}_{k}}
$$

The aim of this section is to demonstrate the error dependencies with respect to element size, the effect of corners, and the effect of uneven sized elements. A two-dimensional model to demonstrate them is shown in Fig. 2. There are two different configurations of boundary elements and nodes:

Even-type configurations: The nodes are placed with even interval, i.e., the size of every element is same.

Uneven-type configurations: By appending two additional nodes to the even-type configuration at the bottom side and adjacent to the upper-right corner, two original elements are replaced by 4 smaller elements, sized half that of original ones. 
The size of standard element, $L$, and the direction, $\phi_{k}$, are changed, but the dimension of the region, $D$, and the wavelength, $\lambda$, are fixed as $D=0.8[\mathrm{~m}], \lambda=0.1[\mathrm{~m}]$. Instead of the element size $L$, the error dependency of element size is shown by $N$, representing the number of elements in a wavelength, i.e., $N=\lambda / L$. Gauss' 4 points quadrature formula $[29,30]$ is applied to the numerical integral for non-singular elements. Notably, we also examined use of 8 points quadrature, but no significant difference was found. This indicates that the error due to numerical integration is sufficiently small compared to the error due to other effects such as truncations of higher derivatives.

In addition, to avoid ambiguity of the direction of the normal unit vector $\boldsymbol{n}$ at the corners, we employed a double node technique with zero distance for the corner nodes $[27,28]$, in which each corner node has three variables; one field $u$, and two normal derivatives for different direction $q_{\gamma}$ and $q_{\gamma^{\prime}}$.

\subsection{Error properties for even-type configuration}

Figure 3 shows the difference vector, $\Delta \boldsymbol{e}_{k}$, with $N=10$ for the even-type configuration for different $\phi_{k}$. It is found that the error at a corner is larger than that on the sides, and its magnitude at such a corner reaches almost $10 \%$ of true solutions.

From the result of $\phi_{k}=0$ symmetrical error with respect to the horizontal axis $\left(\xi_{2}=0\right)$ is found. However, symmetry with respect to the vertical axis $\left(\xi_{1}=0\right)$ cannot be found. It may appear counterintuitive; however, it is not a wrong result. The reason for these results is the difference of the nature between the terms $\left(\nabla_{y} u^{*}\right) q$ and $\left(\nabla_{y} q^{*}\right) u$ in the integrand in Eq. (40). The symmetrical nature of the $\nabla_{y} u^{*}$ and $\nabla_{y} q^{*}$ can be evaluate by Eqs. (13) and (17). In the case where the symmetrical axis is the horizontal axis $\left(\xi_{2}=0\right)$, each the $\left(\nabla_{y} u^{*}\right) \cdot \boldsymbol{e}_{1},\left(\nabla_{y} q^{*}\right) \cdot \boldsymbol{e}_{1}, u$ and $q$ has even symmetry, and each of the $\left(\nabla_{y} u^{*}\right) \cdot \boldsymbol{e}_{2}$, $\left(\nabla_{y} q^{*}\right) \cdot \boldsymbol{e}_{2}$ has odd symmetry. Therefore, the above-mentioned difference in Eq. (40), $\left(\nabla_{y} u^{*}\right) q-\left(\nabla_{y} q^{*}\right) u$, which is the integrand of $\boldsymbol{J}$, has even and odd symmetry for horizontal and vertical component, respectively. In contrast, in the case where the symmetrical axis is the vertical axis $\left(\xi_{1}=0\right)$, although the gradients have similar natures, the natures of $u$ and $q$ have neither even or odd symmetry; $u$ at symmetrical point is equal to the complex conjugate of $u$ at the original point, and in terms of $q$ we have the complex conjugate with opposite sign. Thus, there is no symmetry with respect to the vertical axis. In the 
case where $\phi_{k}=\pi / 4$, shown in the bottom two graphs in Fig. 3, a symmetry with respect to the line of $\xi_{1}=\xi_{2}$ is observed. This is caused by the symmetry of all the quantities of $u, q, \nabla_{y} u^{*}$, and $\nabla_{y} q^{*}$.

Figure 4 shows error dependencies of element size. It is found that the error decreases with increasing $N$, which is equivalent to decrease of the size of element $L$. For almost all of the results the dependency of $N$ obeys a $1 / N$ law, which agrees with the discussions in the section 4 . The significant error is the error due to the non-singular integral $\Delta \boldsymbol{J}_{n_{i}}\{u\}$ shown in Eq. (63). Some exceptions are found in cases where the field node is located around the center of the left-side or right-side for $\phi_{k}=0$, in which the dependency shows $1 / N^{2}$. In these cases, the error due to the truncation of higher order derivatives in the representation of field on the left- or right-hand side of the boundary disappears, because the wave front of the propagation wave is parallel to each side, over which both the $u$ and $q$ are uniform. Therefore, the error $\Delta \boldsymbol{J}_{n_{i}}\{u\}$ from non-singular far element on top- or bottom side, $\left(r_{i}>4 \lambda\right)$, becomes significant. Since the error in Eq. (63) is a consequence of the assumption of $l \ll r_{i} \ll 1 / k$, we can not use the estimation. However, it can be estimated directly by substituting the second of Eq. (61) into Eq. (59). In the case where $\boldsymbol{n}_{i} \cdot \boldsymbol{e}_{\boldsymbol{r}_{i}} \sim 0$, it is $\left|\Delta \boldsymbol{J}_{n_{i}}\{u\}\right|=\frac{(k L)^{2}\left|\sin ^{2} \phi\right|}{24 \beta}\left|u\left(\boldsymbol{x}_{i}\right) \frac{\partial u^{*}}{\partial r}\right|$, which is proportional to $1 / N^{2}$.

We can also draw some conclusions of the errors around corners from the result shown at the right-hand side column of Fig. 4. For the case of sufficiently fine element resolution (e.g., $N=100$ ) the errors at the corner nodes are larger than that atjacent nodes, for which distance from the corner is $L$. It depends on the $\phi_{k}$. In the case of $\phi_{k}=0$ their ratio is almost 4 , and in the case of $\phi_{k}=\pi / 4$ they are almost same. This result agrees with the discussion in the section 4.3 ; i.e., the error at the corner that is induced from the the norm $\left\|\overleftrightarrow{\mathbf{C}^{-1}}\right\|$ grows almost 5 times larger than that on flat boundary in the worst case. The reason why the errors are almost equals in the case of $\phi_{k}=\pi / 4$ can be also explained by previous analysis in terms of symmetry. The growth factor $\overleftrightarrow{\mathbf{C}^{-1}}$, and the maximum norm of $\overleftrightarrow{\mathbf{C}^{-1}}$ is evaluated by the right-hand side of Eq. (99) in Appendix C. If the error vector $\Delta \boldsymbol{J}$ multiplied to $\overleftrightarrow{\mathbf{C}^{-1}}$ is known, the error growth can be estimated precisely using Eq. (98). The significant error at every corner is $\Delta \boldsymbol{J}_{n_{i}}\{u\}$ as 
discussed above, which magnitude and direction are shown in Eqs. (63) and (61), respectively. There are two dominant elements within this error source: namely the two non-singular elements adjoining to two singular elements, where the normal vectors point in different directions. Estimating sum of them for two corners (upper-right and upper-left), we can find that both the absolute magnitudes of error are same but the directions of the error vectors are different each other. The vector component satisfies, respectively, $\Delta \boldsymbol{J} \cdot \boldsymbol{e}_{1}=\Delta \boldsymbol{J} \cdot \boldsymbol{e}_{2}$ and $\Delta \boldsymbol{J} \cdot \boldsymbol{e}_{1}=-\Delta \boldsymbol{J} \cdot \boldsymbol{e}_{2}$ for the upper-right corner and for

the upper-left corner. Using these relations the norm of $\overleftrightarrow{\mathbf{C}^{-1}}$ can be evaluated. The norms at both corners for $\phi_{k}=\pi / 4$ become

$$
\left\|\overleftrightarrow{\mathbf{C}^{-1}}\right\|=\frac{\left\|\overleftrightarrow{\mathbf{C}^{-1}} \cdot \Delta \boldsymbol{J}\right\|}{\|\Delta \boldsymbol{J}\|}=\frac{4 \pi}{\pi+2} \sim 2.4
$$

This result is almost equal to the norm for $\Delta \theta=\pi$, which is equal to 2 as shown in Eq. (68).

\subsection{Error properties for the uneven-type configuration}

Figure 5 shows the effect in the case where smaller sized elements are included. Although the uneven-type configuration uses smaller sized elements, the growth of error is found from a comparison of the zoomed graphs around the replaced elements. The growth is not limited to only this example. The detail results are not shown here, but we have obtained that the amplitude each error obeys $1 / N$ as similar to the case of even-type configuration shown in Fig. 4. It means that the significant error is $\Delta \boldsymbol{J}_{n_{i}}\{u\}$ shown in Eq. (63) even in the case of uneven-type configuration. The error at the additional node on the bottom side is almost twice as large as neighboring ones. Although the element size of the singular element is reduced by appending a new node, the size of the non-singular element $L$ is not changed. However, since $\beta$ appeared in the denominator in the equation is proportional to the distance between the center of the element and the singular node, the $\beta$ does become smaller by appending the new node. It means $\beta=3 / 2$ for the even-type configuration, and $\beta=1$ for the uneven-type configuration. Thus, the error from the non-singular element which connects to the singular elements increases by appending the new node. From Eq. (63), the growth of $\Delta \boldsymbol{J}_{n_{i}}\{u\}$ by appending the new node is estimated as 9/4 times larger than the even-type configuration. 
The error near a corner is similar to the case where the additional node is located on the bottom side. The error at the additional nodes has larger error than that at the adjacent node located opposite to the corner node. At the corner node a little improvement is gained. The dominant error at the corner is caused by the $\Delta \boldsymbol{J}_{n_{i}}\{u\}$ from horizontal side as discussed in the previous subsection. Since the element with the most significant error is the one adjacent to the additional node that is opposite to the corner, the element size becomes half of the size of the original element and $\beta$ is same as the original configuration. Thus, the error from this element decreases. Note that the error is not reduced to exactly half, since the error from the other elements is not reduced.

From the above discussion we can conclude that when the configuration contains uneven sized elements even if their sizes are finer than the other even sized elements, it may still result in larger error around them.

\subsection{Error reduction around the corner}

As discussed above, the errors at corners are generally larger than at other nodes. Furthermore, replacement of an element around corner to several smaller sized elements induces a larger error in the areas around the corners. However, it does afford a little improvement exactly on the corner nodes. To reduce the error at the corner nodes, we evaluated two configurations. One is a gradual size-variation configuration. In this configuration, the element adjacent to the corner node on either side is replaced by four smaller elements with a quarter size of the original element; moreover, the next consecutive element following this replaced element is also replaced two half-sized elements. In the other configuration the two elements adjacent to the corner node are replaced by eight quarter-sized elements on each side, i.e., the number of new fine elements is sixteen. Both of these results are shown in Fig. 6, together with the result of the original even-type configuration. The results of both new configurations demonstrate the error reduction at the corner. However, the errors at the additional nodes are relatively larger than the errors on the side near the corner. The growth of the error on the side in the case of gradual size varying is smaller than that of the replacement by eight quarter-sized elements on each side. These results agree with the results discussed in the above subsections. 


\section{Conclusion}

This paper shows the regularization of the hypersingular term that appears in the gradient representation of a boundary integral equation for a 2-dimensional Helmholtz equation. Since this regularization is achieved by an analytical approach and it does not use the double integral technique [2], a computational cost may be significantly reduced. Moreover, this regularization is applicable even if the nodes include corner locations or if the size of elements is non-uniform. This paper also shows that the error from this regularization in terms of linear elements is estimated roughly by separation into the error from the singular elements and that from non-singular elements. The computational result from evaluated examples demonstrates that the calculated errors are in agreement with the roughly estimated error. The error caused by the hypersingular element regularized in the proposed method is negligibly small in comparison to the non-singular element. The dominant non-singular error decreases with increasing of the number of elements, $N$, such that the total error is proportional to $1 / N$. When some nodes are modified by appendage of evenly sized boundary elements, the properties of the error vary according to the location of the appended nodes. In the case where the additional node is located on a flat boundary, in which the size of two adjacent boundary elements to the node are different, the error increases in spite of the reduced boundary size. In the case where the additional node is located in the vicinity of a corner, the error at the corner decreases, but the error at adjacent nodes along a flat boundary increases. In order to reduce the error effectively by the regularization technique proposed in this paper, it is recommended to use even sized elements for flat boundaries, and for corner areas to employ a configuration of gradual variation of element sizes.

\section{A. Representation of the second order derivative of the field at the singular point}

The purpose of this appendix is to reformulate the two second order derivatives in Eq. (28) as the linear combination of $u$ and $q$ at the singular point $y$ or at adjacent nodes.

The two variables that should be represented finally are $\left.\frac{\partial^{2} u}{\partial \tau_{\gamma} n_{\gamma}}\right|_{y}$ and $\left.\frac{\partial^{2} u}{\partial \tau_{\gamma}^{2}}\right|_{y}$ where $\gamma$ is $a$ or $b$ and the unit vectors $\boldsymbol{n}_{\gamma}, \boldsymbol{\tau}_{\gamma}$ are shown in Fig. 1. Let us label $\gamma^{\prime}$ which expresses associated quantities with the opposite side of the boundary $\Gamma_{\gamma}$ to $\boldsymbol{y}$. Notice that $\left(\gamma, \gamma^{\prime}\right)$ 
can be taken as either pair $(a, b)$, or $(b, a)$; however, their identification is not important because no quantities dependent on the difference between them are found in the following formulation.

We assume the second derivative of $u$ is defined at a point $\boldsymbol{x}$ located on each boundary element, and derivatives of third or higher orders are negligibly small. Accordingly, the Taylor expansion around $\boldsymbol{y}$ can be given as follows.

$$
\begin{aligned}
u(\boldsymbol{x}) \sim u(\boldsymbol{y}) & +(\boldsymbol{x}-\boldsymbol{y}) \cdot(\nabla u)_{\boldsymbol{y}} \\
& +\frac{1}{2}(\boldsymbol{x}-\boldsymbol{y}) \cdot(\boldsymbol{x}-\boldsymbol{y}) \cdot(\nabla \nabla u)_{\boldsymbol{y}} .
\end{aligned}
$$

Since the quantity $q$ has been already applied to the differential operator, the Taylor expansion is represented up to the first order.

$$
q_{\gamma}(\boldsymbol{x}) \sim q_{\gamma}(\boldsymbol{y})+(\boldsymbol{x}-\boldsymbol{y}) \cdot\left(\nabla q_{\gamma}\right)_{\boldsymbol{y}}
$$

where $q_{\gamma}=\nabla u \cdot \boldsymbol{n}_{\gamma}$.

The relation between the pairs of unit vectors $\left(\boldsymbol{\tau}_{\gamma}, \boldsymbol{n}_{\gamma}\right)$ and $\left(\boldsymbol{\tau}_{\gamma^{\prime}}, \boldsymbol{n}_{\gamma^{\prime}}\right)$ are represented by the internal angle $\Delta \theta$ at the singular point $\boldsymbol{y}$. The following inner products are corresponding to the components of the coordinate transform matrix,

$$
\begin{array}{ll}
T_{\tau^{\prime} \tau} \triangleq \boldsymbol{\tau}_{\gamma^{\prime}} \cdot \boldsymbol{\tau}_{\gamma}=\cos \Delta \theta, & T_{\tau^{\prime} n} \triangleq \boldsymbol{\tau}_{\gamma^{\prime}} \cdot \boldsymbol{n}_{\gamma}=-\sin \Delta \theta, \\
T_{n^{\prime} \tau} \triangleq \boldsymbol{n}_{\gamma^{\prime}} \cdot \boldsymbol{\tau}_{\gamma}=-\sin \Delta \theta, & T_{n^{\prime} n} \triangleq \boldsymbol{n}_{\gamma^{\prime}} \cdot \boldsymbol{n}_{\gamma}=-\cos \Delta \theta .
\end{array}
$$

The derivative operators can be transformed by the coordinate transform matrix between $\left(\tau_{\gamma}, n_{\gamma}\right)$ system and $\left(\tau_{\gamma^{\prime}}, n_{\gamma^{\prime}}\right)$ given by the following derivative operators.

$$
\left(\begin{array}{l}
\partial_{\tau^{\prime}} \\
\partial_{n^{\prime}}
\end{array}\right)=\left(\begin{array}{c}
T_{\tau^{\prime} \tau} T_{\tau^{\prime} n} \\
T_{n^{\prime} \tau} T_{n^{\prime} n}
\end{array}\right)\left(\begin{array}{l}
\partial_{\tau} \\
\partial_{n}
\end{array}\right)=\left(\begin{array}{c}
T_{\tau^{\prime} j} \partial_{j} \\
T_{n^{\prime} j} \partial_{j}
\end{array}\right),
$$

where the notation of $\partial_{\alpha}$ is an abbreviation of $\frac{\partial}{\partial \alpha}$. Moreover, the summation symbol with respect to $j$ for $\tau, n$ is omitted in the final notation.

The position vectors $\boldsymbol{x}_{\gamma}$ and $\boldsymbol{x}_{\gamma^{\prime}}$ are expressed by the unit tangential vectors $\boldsymbol{\tau}_{\gamma}$ of $\Gamma_{\gamma}$, as well as the vectors $\tau_{\gamma^{\prime}}$ of $\Gamma_{\gamma^{\prime}}$,

$$
\boldsymbol{x}_{\gamma}-\boldsymbol{y}=L_{\gamma} \boldsymbol{\tau}_{\gamma}, \quad \boldsymbol{x}_{\gamma^{\prime}}-\boldsymbol{y}=L_{\gamma^{\prime}} \boldsymbol{\tau}_{\gamma^{\prime}},
$$


where $L_{\gamma}$ and $L_{\gamma^{\prime}}$ denote the size of $\Gamma_{\gamma}$ and $\Gamma_{\gamma^{\prime}}$, respectively.

The result of expansion of $u\left(\boldsymbol{x}_{\gamma}\right)$ can be written simply as

$$
u\left(\boldsymbol{x}_{\gamma}\right) \sim u(\boldsymbol{y})+L_{\gamma}\left(\partial_{\tau} u\right)_{\boldsymbol{y}}+\frac{L_{\gamma}^{2}}{2}\left(\partial_{\tau \tau}^{2} u\right)_{\boldsymbol{y}}
$$

The second derivative at the last term is the variable to be solved, while both $u\left(\boldsymbol{x}_{\gamma}\right)$ and $u(y)$ are the variables in the final expression of a linear combination.

In the case of $u\left(\boldsymbol{x}_{\gamma^{\prime}}\right)$ the derivative operator is replaced by the transform matrix in Eq. (76), and then the expansion is written as,

$$
\begin{aligned}
u\left(\boldsymbol{x}_{\gamma^{\prime}}\right) & \sim u(\boldsymbol{y})+L_{\gamma^{\prime}}\left(\partial_{\tau^{\prime}} u\right)_{\boldsymbol{y}}+\frac{L_{\gamma^{\prime}}^{2}}{2}\left(\partial_{\tau^{\prime} \tau^{\prime}}^{2} u\right)_{\boldsymbol{y}} \\
= & u(\boldsymbol{y})+L_{\gamma^{\prime}}\left(T_{\tau^{\prime} \tau}\left(\partial_{\tau} u\right)_{\boldsymbol{y}}+T_{\tau^{\prime} n}\left(\partial_{n} u\right)_{y}\right) \\
+ & \frac{L_{\gamma^{\prime}}^{2}}{2}\left(T_{\tau^{\prime} \tau}^{2}\left(\partial_{\tau \tau}^{2} u\right)_{\boldsymbol{y}}+2 T_{\tau^{\prime} \tau} T_{\tau^{\prime} n}\left(\partial_{\tau n}^{2} u\right)_{y}+T_{\tau^{\prime} n}^{2}\left(\partial_{n n}^{2} u\right)_{y}\right)
\end{aligned}
$$

In this equation the variable $\left(\partial_{n} u\right)_{y}$ is identical to $q_{\gamma}(\boldsymbol{y})$,

$$
\left(\partial_{n} u\right)_{y}=q_{\gamma}(y)
$$

Taking a tangential derivative of this equation, we obtain the other second derivative $\left(\partial_{\tau n}^{2} u\right)_{y}$ that is one of the variable to be represented finally,

$$
\left(\partial_{\tau n}^{2} u\right)_{\boldsymbol{y}}=\left.\frac{\partial^{2} u}{\partial \tau_{\gamma} n_{\gamma}}\right|_{\boldsymbol{y}}=\left.\frac{\partial q_{\gamma}}{\partial \tau_{\gamma}}\right|_{\boldsymbol{y}} \sim \frac{q_{\gamma}\left(\boldsymbol{x}_{\gamma}\right)-q_{\gamma}(\boldsymbol{y})}{L_{\gamma}} .
$$

The relation between the quantities $\left(\partial_{n n}^{2} u\right)_{y}$ and $\left(\partial_{\tau \tau}^{2} u\right)_{y}$ can be given by a Helmholtz equation, because the sum of these quantities equals the Laplacian,

$$
\left(\partial_{n n}^{2} u\right)_{y}+\left(\partial_{\tau \tau}^{2} u\right)_{y}+k^{2} u(y)=0
$$

Eliminating the derivatives except $\left(\partial_{\tau \tau}^{2} u\right)_{y}$ from five independent equations Eqs. (78)-(82), we can represent the $\left(\partial_{\tau \tau}^{2} u\right)_{y}$ using a linear combination form,

$$
\left(\partial_{\tau \tau}^{2} u\right)_{y}=\left.\frac{\partial^{2} u}{\partial \tau_{\gamma}^{2}}\right|_{y}=\sum_{i} W_{\gamma}^{f_{i}} f_{i},
$$

where $f_{i}$ denotes one of the quantities $u$ and $q_{\gamma}$ at $\boldsymbol{y}, \boldsymbol{x}_{\gamma}$ and $\boldsymbol{x}_{\gamma^{\prime}}$,

$$
f_{i} \in\left\{u(\boldsymbol{y}), u\left(\boldsymbol{x}_{\gamma}\right), u\left(\boldsymbol{x}_{\gamma^{\prime}}\right), q_{\gamma}(\boldsymbol{y}), q_{\gamma}\left(\boldsymbol{x}_{\gamma}\right)\right\},
$$


and, each factor for $f_{i}$ is obtained as follows.

$$
\begin{aligned}
& \left(\begin{array}{l}
W_{\gamma}^{u\left(\boldsymbol{x}_{\gamma}\right)} \\
W_{\gamma}^{u(\boldsymbol{y})} \\
W_{\gamma}^{u\left(\boldsymbol{x}_{\gamma^{\prime}}\right)} \\
W_{\gamma}^{q_{\gamma}\left(\boldsymbol{x}_{\gamma}\right)} \\
W_{\gamma}^{q_{\gamma}(\boldsymbol{y})}
\end{array}\right)=\frac{1}{D}\left(\begin{array}{l}
-2 L_{\gamma^{\prime}} \cos \Delta \theta \\
+2 L_{\gamma^{\prime}} \cos \Delta \theta-2 L_{\gamma}\left(1-\frac{\left(k L_{\gamma^{\prime}}\right)^{2}}{2} \sin ^{2} \Delta \theta\right) \\
+2 L_{\gamma} \\
+L_{\gamma^{\prime}}^{2} \sin 2 \Delta \theta \\
-L_{\gamma^{\prime}}^{2} \sin 2 \Delta \theta+2 L_{\gamma} L_{\gamma^{\prime}} \sin \Delta \theta
\end{array}\right), \\
& D=L_{\gamma} L_{\gamma^{\prime}}\left(L_{\gamma^{\prime}} \cos 2 \Delta \theta-L_{\gamma} \cos \Delta \theta\right) .
\end{aligned}
$$

These factors depend on the quantity $\Delta \theta$ as well as the sizes of elements.

\section{B. Analytical representation of integrals appeared in the singular element}

The purpose of this appendix is to show the analytical representation of the two integrals in Eq. (28). A numerical integral is obtainable because these integrals are regular or weakly singular. However, analytical representation is useful to improve the accuracy and to understand the nature of the integrals.

The second integral in Eq. (28) is transformed to the following relation by partial integration,

$$
\begin{aligned}
\int_{0}^{L_{\gamma}} r \frac{\partial u^{*}}{\partial r} d r & =\left[r u^{*}\right]_{r=L_{\gamma}}-\int_{0}^{L_{\gamma}} u^{*} d r \\
& =\frac{L_{\gamma}}{4 j} H_{0}^{(2)}\left(k L_{\gamma}\right)-\frac{1}{4 j} \int_{0}^{L_{\gamma}} H_{0}^{(2)}(k r) d r
\end{aligned}
$$

where the second term has a weakly singularity. This integration is given by Struve function, $\mathbf{H}_{n}(x)[30]$,

$$
\begin{aligned}
\int_{0}^{x} H_{0}^{(2)}(x) d x= & x H_{0}^{(2)}(x) \\
& +\frac{\pi x}{2}\left(\mathbf{H}_{0}(x) H_{1}^{(2)}(x)-\mathbf{H}_{1}(x) H_{0}^{(2)}(x)\right) .
\end{aligned}
$$

Since the first term of the integral of Eq. (86) and the first term of Eq. (87) are canceled, the integral of Eq. (86) is reduced to

$$
\begin{aligned}
\int_{0}^{L_{\gamma}} r \frac{\partial u^{*}}{\partial r} d r=\frac{j \pi L_{\gamma}}{8} & \left(\mathbf{H}_{0}\left(k L_{\gamma}\right) H_{1}^{(2)}\left(k L_{\gamma}\right)\right. \\
& \left.-\mathbf{H}_{1}\left(k L_{\gamma}\right) H_{0}^{(2)}\left(k L_{\gamma}\right)\right) .
\end{aligned}
$$


The integer order of the Hankel function, $H_{n}^{(2)}(x)$, and the Struve function, $\mathbf{H}_{n}(x)$, are given by the following infinite series.

$$
\begin{aligned}
H_{n}^{(2)}(x)= & J_{n}(x)-j Y_{n}(x), \\
J_{n}(x)= & \left(\frac{x}{2}\right)^{n} \sum_{m=0}^{\infty} a_{n m}\left(\frac{x}{2}\right)^{2 m}, \\
Y_{n}(x)= & \frac{-1}{\pi} \sum_{m=0}^{n-1} \frac{(n-m-1) !}{m !}\left(\frac{x}{2}\right)^{2 m-n}+\frac{2}{\pi} \log \frac{x}{2} J_{n}(x) \\
& -\frac{1}{\pi} \sum_{m=0}^{\infty}\left(\psi_{m+1}+\psi_{n+m+1}\right) a_{n m}\left(\frac{x}{2}\right)^{2 m+n}, \\
a_{n m}= & \frac{(-1)^{m}}{m !(n+m) !}, \quad \psi_{m+1}=-\gamma+\sum_{l=1}^{m} \frac{1}{l}, \\
\mathbf{H}_{n}(x)= & \left(\frac{x}{2}\right)^{n+1} \sum_{m=0}^{\infty} \frac{(-1)^{m}}{\Gamma\left(m+\frac{3}{2}\right) \Gamma\left(n+m+\frac{3}{2}\right)}\left(\frac{x}{2}\right)^{2 m},
\end{aligned}
$$

where $\gamma$ is the Euler constant, and $\Gamma$ represents the Gamma function. In the case of $k L_{\gamma} \ll 1$ the infinite series converges rapidly. The integral around $k L_{\gamma} \sim 0$ can be approximated as

$$
\int_{0}^{L_{\gamma}} r \frac{\partial u^{*}}{\partial r} d r \sim \frac{-L_{\gamma}}{2 \pi}, \quad\left(k L_{\gamma} \sim 0\right)
$$

Similarly, the other integral in Eq. (28) that is a weakly singular integral can be represented without any singularity using Eqs. (89)-(92),

$$
\begin{aligned}
\int_{0}^{L_{\gamma}} & \frac{\partial}{r} \frac{\delta u^{*}}{\partial r} d r=\int_{0}^{L_{\gamma}} \frac{1}{r}\left(\frac{-k}{4 j} H_{1}^{(2)}(k r)-\frac{-1}{2 \pi r}\right) d r \\
= & \frac{-k}{4 j} \sum_{m=0}^{\infty} \frac{a_{1 m}}{2 m+1}\left(\frac{k L_{\gamma}}{2}\right)^{2 m+1} \\
& \cdot\left(1+\frac{j}{\pi}\left(\psi_{m+1}+\psi_{m+2}+\frac{1}{m+\frac{1}{2}}-2 \log \frac{k L_{\gamma}}{2}\right)\right) .
\end{aligned}
$$

\section{Inverse matrix of the coefficient matrix}

To ensure the existence of an inverse matrix of $\overleftrightarrow{\mathbf{C}}$ the determinant of $\overleftrightarrow{\mathbf{C}}$ must not be 0 . The evaluation of the determinant is shown in this appendix. Both the differences 
$\underset{\gamma: a-b}{\operatorname{Diff}}\left[\sin 2 \theta_{\gamma}\right]$ and $\underset{\gamma: a-b}{\operatorname{Diff}}\left[\cos 2 \theta_{\gamma}\right]$ in the definition of $\overleftrightarrow{\mathbf{C}}$ (Eq. (41)) are reduced to the following relation,

$$
\left(\begin{array}{c}
\operatorname{Diff}\left[\sin 2 \theta_{\gamma}\right] \\
\underset{\gamma: a-b}{\operatorname{Diff}}\left[\cos 2 \theta_{\gamma}\right]
\end{array}\right)=2 \sin \Delta \theta\left(\begin{array}{c}
\cos 2 \theta_{0} \\
-\sin 2 \theta_{0}
\end{array}\right), \quad \theta_{0}=\frac{\theta_{a}+\theta_{b}}{2} .
$$

Using this relation the determinant of $\overleftrightarrow{\mathbf{C}}$ is represented as

$$
\begin{aligned}
\operatorname{det} \overleftrightarrow{\mathbf{C}} & =\frac{1}{4 \pi^{2}}\left((\Delta \theta)^{2}-\sin ^{2} \Delta \theta\right)+\left(\operatorname{Diff}_{\gamma: a-b}\left[u^{*}\left(L_{\gamma}\right)\right]\right)^{2} \\
= & \frac{1}{4 \pi^{2}}\left((\Delta \theta)^{2}-\sin ^{2} \Delta \theta\right) \\
& +\left\{\left(\mathfrak{R}\left\{\operatorname{Diff}_{\gamma: a-b}\left[u^{*}\left(L_{\gamma}\right)\right]\right\}\right)^{2}-\left(\mathfrak{J}\left\{\operatorname{Diff}_{\gamma: a-b}\left[u^{*}\left(L_{\gamma}\right)\right]\right\}\right)^{2}\right\} \\
& +2 j \mathfrak{R}\left\{\operatorname{Difff}_{\gamma: a-b}\left[u^{*}\left(L_{\gamma}\right)\right]\right\} \mathfrak{J}\left\{\operatorname{Difff}_{\gamma: a-b}\left[u^{*}\left(L_{\gamma}\right)\right]\right\} .
\end{aligned}
$$

The first and second terms on the right-hand side are real numbers. In contrast, the last term is a pure imaginary number. In order to satisfy the condition that the determinant is not equal to 0 , either the real or the imaginary part must have a non-zero value. The first term is always positive. The second term depends on $L_{\gamma}$. In general, the element size is chosen as a size sufficiently small compared to the wave length, i.e., $L_{\gamma} \ll 1 / k$. In this case the fundamental solution $u^{*}\left(L_{\gamma}\right)$ has a larger real part than its imaginary part; therefore, the second term has also a positive real number. Since the real part of the determinant given by the sum of the first term and the second term is a positive number, hence the determinant has a non-zero value and we can conclude that $\overleftrightarrow{\mathbf{C}}$ has an inverse matrix in the case $L_{\gamma} \ll 1 / k$.

The inverse matrix $\overleftrightarrow{\mathbf{C}^{-1}}$ can be readily obtained from Eq. (41) and Eq. (96) as follows:

$$
\begin{aligned}
\overleftrightarrow{\mathbf{C}^{-1}}=\frac{1}{\operatorname{det} \overleftrightarrow{\mathbf{C}}}\left[\left(\begin{array}{cc}
\frac{\Delta \theta}{2 \pi} & -\underset{\gamma: a-b}{\operatorname{Diff}}\left[u^{*}\left(L_{\gamma}\right)\right] \\
+\underset{\gamma: a-b}{\operatorname{Diff}}\left[u^{*}\left(L_{\gamma}\right)\right] & \frac{\Delta \theta}{2 \pi}
\end{array}\right)\right. \\
\left.+\frac{\sin \Delta \theta}{2 \pi}\left(\begin{array}{ll}
-\cos 2 \theta_{0} & -\sin 2 \theta_{0} \\
-\sin 2 \theta_{0} & +\cos 2 \theta_{0}
\end{array}\right)\right]
\end{aligned}
$$


Since the second matrix in the square bracket is an orthogonal matrix, the second order norm becomes unity. Taking a maximum norm for the first matrix, we can obtain the following relation.

$$
\left\|\overleftrightarrow{\mathbf{C}^{-1}}\right\| \leq \frac{1}{|\operatorname{det} \overleftrightarrow{\mathbf{C}}|}\left(\frac{|\Delta \theta|+|\sin \Delta \theta|}{2 \pi}+\left|\operatorname{Diff}_{\gamma: a-b}\left[u^{*}\left(L_{\gamma}\right)\right]\right|\right)
$$

\section{Error due to truncated terms of the singular element}

\section{D.1. In the case of the node on flat boundary}

Since the second order derivatives of $u$ in Eqs. (44) and (45) are represented using the quantities $u$ and $q$ at the singular node $\boldsymbol{y}$, as well as adjacent nodes, $\boldsymbol{x}_{\gamma}$ and $\boldsymbol{x}_{\gamma^{\prime}}$, as shown in Appendix A, the third or higher derivatives cause error of truncation. To estimate the error due to the truncation we must estimate the third derivative. However, since there is a case where the third derivative vanishes under a certain condition, we also consider the fourth derivative.

In the case of a flat boundary $(\Delta \theta=\pi)$, the factors of coordinate transform matrix in Eq. (76) are given by Eq. (75) as follows:

$$
T_{\tau^{\prime} \tau}=-1, \quad T_{n^{\prime} n}=+1, \quad T_{\tau^{\prime} n}=T_{n^{\prime} \tau}=0 .
$$

From these factors the fourth order of the Taylor expansions at the nodes, $\boldsymbol{x}_{\gamma}$ and $\boldsymbol{x}_{\gamma^{\prime}}$, around the singular node, $\boldsymbol{y}$, are obtained as follows:

$$
\begin{aligned}
u\left(\boldsymbol{x}_{\gamma}\right) \sim u(\boldsymbol{y}) & +L\left(\partial_{\tau} u\right)_{\boldsymbol{y}}+\frac{L^{2}}{2}\left(\partial_{\tau \tau}^{2} u\right)_{\boldsymbol{y}} \\
& +\frac{L^{3}}{6}\left(\partial_{\tau \tau \tau}^{3} u\right)_{\boldsymbol{y}}+\frac{L^{4}}{24}\left(\partial_{\tau \tau \tau \tau}^{4} u\right)_{\boldsymbol{y}}, \\
u\left(\boldsymbol{x}_{\gamma^{\prime}}\right) \sim u(\boldsymbol{y})- & \alpha L\left(\partial_{\tau} u\right)_{\boldsymbol{y}}+\frac{(\alpha L)^{2}}{2}\left(\partial_{\tau \tau}^{2} u\right)_{\boldsymbol{y}} \\
& -\frac{(\alpha L)^{3}}{6}\left(\partial_{\tau \tau \tau}^{3} u\right)_{\boldsymbol{y}}+\frac{(\alpha L)^{4}}{24}\left(\partial_{\tau \tau \tau}^{4} u\right)_{y},
\end{aligned}
$$

where sizes of elements are set as $L_{\gamma}=L$ and $L_{\gamma^{\prime}}=\alpha L$. Eliminating $\left(\partial_{\tau} u\right)_{y}$ and 
rearranging with respect to $\left(\partial_{\tau \tau}^{2} u\right)_{y}$, we can obtain the following representation of $\left(\partial_{\tau \tau}^{2} u\right)_{y}$.

$$
\begin{aligned}
& \left(\partial_{\tau \tau}^{2} u\right)_{\boldsymbol{y}} \sim \frac{2\left(\alpha u\left(\boldsymbol{x}_{\gamma}\right)-(1+\alpha) u(\boldsymbol{y})+u\left(\boldsymbol{x}_{\gamma^{\prime}}\right)\right)}{\alpha(1+\alpha) L^{2}}+\Delta_{\partial_{\tau \tau}^{2} u}^{(3)}+\Delta_{\partial_{\tau \tau}^{2} u}^{(4)}, \\
& \Delta_{\partial_{\tau \tau}^{2} u}^{(3)} \triangleq-\frac{L(1-\alpha)}{3}\left(\partial_{\tau \tau \tau}^{3} u\right)_{y}, \\
& \Delta_{\partial_{\tau \tau}^{2} u}^{(4)} \triangleq-\frac{L^{2}\left(1+\alpha^{3}\right)}{12(1+\alpha)}\left(\partial_{\tau \tau \tau \tau}^{4} u\right)_{y},
\end{aligned}
$$

where the variable shown as $\Delta_{f}^{(n)}$ represents the truncated term for some function $f$ associated with $n$-th order of derivative. In the case of $\alpha=1$, the error due to the third derivative vanishes and the fourth one becomes dominant.

In contrast, the derivative of $q$ is simpler because it can be given by only the Taylor expansion at $u\left(\boldsymbol{x}_{\gamma}\right)$. The Taylor expansion of $q$ using third derivative of $u$, which is equivalent to the second derivative of $q$, is given as

$$
q_{\gamma}\left(\boldsymbol{x}_{\gamma}\right) \sim q_{\gamma}(\boldsymbol{y})+L\left(\partial_{\tau} q_{\gamma}\right)_{\boldsymbol{y}}+\frac{L^{2}}{2}\left(\partial_{\tau \tau}^{2} q_{\gamma}\right)_{\boldsymbol{y}}
$$

where $q_{\gamma}=\nabla u \cdot \boldsymbol{n}_{\gamma}$. Rearranging about $\left(\partial_{\tau} q_{\gamma}\right)_{\boldsymbol{y}}$, we can estimate the error of truncation of $q_{\gamma}$ as follows:

$$
\begin{aligned}
& \left(\partial_{\tau} q_{\gamma}\right)_{\boldsymbol{y}} \sim \frac{q_{\gamma}\left(\boldsymbol{x}_{\gamma}\right)-q_{\gamma}(\boldsymbol{y})}{L}+\Delta_{\partial_{\tau n}^{2} u}^{(3)} \\
& \Delta_{\partial_{\tau n}^{2} u}^{(3)} \triangleq-\frac{L}{2}\left(\partial_{\tau \tau}^{2} q_{\gamma}\right)_{\boldsymbol{y}}=-\frac{L}{2}\left(\partial_{\tau \tau n}^{3} u\right)_{\boldsymbol{y}} .
\end{aligned}
$$

D.2. In the case of the node at a corner with a right angle

This case $(\Delta \theta=\pi / 2)$ is simple because the third derivative of $u$ is not canceled. Since the factors of geometry are given as

$$
T_{\tau^{\prime} \tau}=T_{n^{\prime} n}=0, \quad T_{\tau^{\prime} n}=T_{n^{\prime} \tau}=-1,
$$

the Taylor expansion of $u$ at $\boldsymbol{x}_{\gamma^{\prime}}$ is given as follows:

$$
\begin{gathered}
u\left(\boldsymbol{x}_{\gamma^{\prime}}\right) \sim u(\boldsymbol{y})- \\
-\alpha L\left(\partial_{n} u\right)_{y}+\frac{(\alpha L)^{2}}{2}\left(\partial_{n n}^{2} u\right)_{y} \\
-\frac{(\alpha L)^{3}}{6}\left(\partial_{n n n}^{3} u\right)_{y} .
\end{gathered}
$$


The normal derivative $\left(\partial_{n} u\right)_{y}$ at the second term is identical to $q_{\gamma}(y)$, and also $\left(\partial_{n n}^{2} u\right)_{y}$ at the third term can be represented by $\left(\partial_{\tau \tau}^{2} u\right)_{y}$ using Eq. (82). Consequently, $\left(\partial_{\tau \tau}^{2} u\right)_{y}$ is reduced as follows:

$$
\begin{aligned}
& \left(\partial_{\tau \tau}^{2} u\right)_{\boldsymbol{y}} \sim \frac{2\left(\left(1-\frac{(k \alpha L)^{2}}{2}\right) u(\boldsymbol{y})-u\left(\boldsymbol{x}_{\gamma^{\prime}}\right)-\alpha L q_{\gamma}(\boldsymbol{y})\right)}{(\alpha L)^{2}}+\Delta_{\partial_{\tau \tau}^{2} u}^{(3)} \\
& \left(\partial_{\tau \tau}^{2} u\right)_{\boldsymbol{y}} \sim \Delta_{\partial_{\tau \tau}^{2} u}^{(3)} \triangleq-\frac{(\alpha L)}{3}\left(\partial_{n n n}^{3} u\right)_{\boldsymbol{y}} .
\end{aligned}
$$

In terms of the tangential derivative of $q_{\gamma}$ we can derive the same relation to that in the case of the flat boundary shown in Eq. (107). 


\section{References}

[1] Schenck HA. Improved integral formulation for acoustic radiation problems. J. Acoustical Soc. America 1968; 44(1):41-58.

[2] Burton AJ. Miller GF. The application of integral equation methods to the numerical solution of some exterior boundary-value problems. Proc. Royal Society of London. Series A 1971; 323:201-210

[3] Ursell F. On the exterior problems of acoustics. Proc. Cambridge Philosophical Society $1973 ; 74: 117-125$.

[4] Tomioka S. Enoto T. Removal of spurious solutions in boundary element method analysis for Fabry-Perot resonator containing another medium. Trans. Institute of Electrical Engineers of Japan 1993; 113-A(8):572-579 (Japanese).

[5] Tomioka S. Enoto T. Fukai I. Removing spurious solutions in boundary elemental method for multimedia problems using virtual boundary dividing method. Int. J. of Applied Electromagnetics in Materials 1994; Suppl. 5:357-360.

[6] Chen JT. Chen IL. Chen KH. Treatment of rank-deficiency in acoustics using SVD. J. Comp. Acoustics 2006; 14(2):157-183.

[7] Tanaka M. Sladek V. Sladek J. Regularization techniques applied to boundary element method. Appl. Mech. Reviews 1994; 47(10):457-499.

[8] Chen JT. Hong H-K. Review of dual boundary element methods with emphasis on hypersingular integrals and divergent series. Appl. Mech. Reviews 1999; 52(1):17-33.

[9] Hwang WS. Hypersingular boundary integral equations for exterior acoustic problems. J. Acoust. Soc. Am. 1997; 101(6):3336-3342.

[10] Yang SA. Evaluation of 2-D Green's boundary formula and its normal derivative using Legendre polynomials, with an application to acoustic scattering problems. Int. J. Numerical Method in Engineering 2002; 53(4):905-27. 
[11] Yan ZY. Hung KC. Zheng H. Solving the hypersingular boundary integral equation in three-dimensional acoustics using a regularization relationship. J. Acoust. Soc. Am. 2003; 113(5):2674-2683.

[12] Mitzner KM. Acoustic scattering from an interface between media of greatly different density. J. Mathematical Physics 1966; 7:2053-2060.

[13] Stallybrass MP. On a pointwise variational principle for the approximate solution of linear boundary value problems. J. Mathematics and Mechanics 1967; 16(11):1247-1286.

[14] Meyer WL. Bell WA. Zinn BT. Boundary integral solutions of three dimensional acoustic radiation problems. J. Sound and Vibration 1978; 59(2):245-262.

[15] Terai T, On calculation of sound fields around three dimensional objects by integral equation methods. J. Sound and Vibration 1980; 69(1):71-100.

[16] Mathews IC. Numerical techniques for three-dimensional steady-state fluid-structure interaction. J. Acoust. Soc. Am. 1986; 79(5):1317-1325.

[17] Krishinasamy G. Schmerr LW. Rudolphi TJ. Rizzo FJ. Hypersingular boundary integral equations: Some applications in acoustic and elastic wave scattering. Trans. ASME:J. Appl. Mech. 1990; 57:404-414.

[18] Chien CC. Rajiyah H. Atluri SN. An effective method for solving the hypersingular integral equations in 3-D acoustics. J. Acoust. Soc. Am. 1990; 88(2):918-937.

[19] Wu TW. Seybert AF. Wan GC. On the numerical implementation of Cauchy principal value integral to insure a unique solution for acoustic radiation and scattering. J. Acoust. Soc. Am. 1991; 90(1):554-560.

[20] Liu Y. Chen S. A new form of hypersingular boundary integral equation for 3-D acoustics and its implementation with $C^{0}$ boundary elements. Comput. Methods Appl. Mech. Engrg. 1999; 173:375-386. 
[21] Morse PM. Feshbach H. Methods of theoretical physics. New York : McGraw-Hill; 1953; Chap. 7.

[22] Arai M. Adachi T. Matsumoto H. Highly accurate analysis by boundary element method based on uniform gradient condition. Trans. of the Japan Society of Mechanical Engineers. A 1995; 61(581):161-168 (Japanese).

[23] Guiggiani M. Krishinasamy G. Rudolphi TJ. Rizzo FJ. A general algorithm for the numerical solution of hypersingular boundary integral equations. Trans.

ASME:J. Appl. Mech. 1992; 59:604-614.

[24] Chen IL. Liang MT. Kuo SR. Chen JT. Dual Boundary Integral Equations for Helmholtz equation at a Corner Using Contour Approach around Singularity. J. Marine Sci. and Tech. 2001; 9(1):53-63.

[25] Kisu H. Kawahara T, Boundary element analysis system based on a formulation with relative quantity. In: Brebbia CA. Mathematical and computational aspects (Boundary elements X ; vol. 1). Computational Mechanics: Southampton, 1988; 111-112.

[26] Chen JT. Hong H-K. Dual boundary integral equations at a corner using contour approach around singularity. Advances in Engineering Software, 21(3) 1994; 169-178.

[27] Mitra AK. Ingber MS. Resolving difficulties in the BIEM caused by geometric corners and discontinuous boundary conditions. In: Brebbia CA. Wendland WL. Kuhn G, editors. Mathematical and computational aspects (Boundary elements IX ; vol. 1). Springer-Verlag: Berlin,Tokyo, 1987; 519-532.

[28] Kost A. Shen JX. Treatment of singularities in the computation of magnetic fields with periodic boundary conditions by the boundary element method. IEEE Trans. on Magnetics 1990; 26(2):607-609.

[29] Brebbia CA. Walker S. Boundary element techniques in engineering. London, Boston: Newnes-Butterworths; 1980. 
[30] Abramowitz M. Stegun IA, editors. Handbook of mathematical functions with formulas, graphs, and mathematical tables. Dover: New York; 1972. 


\section{List of Figures}

Figure 1 Boundary excluding the singular point.

Figure 2 Node placements for Even-type and Uneven-type configurations: The filled circles show boundary nodes for the Even-type configuration. For the Uneven-type configuration, in which the interval of nodes are uneven, two additional nodes shown by diamond shaped symbols at the bottom side and around upper right corner are appended. The vector $\boldsymbol{k}$ shows the propagation vector.

Figure 3 Complex error vector $\Delta \boldsymbol{e}_{k}$ of the Even-Type configuration $(N=10)$ : $\quad$ Each pair of figures in a row denotes a set of results for $\phi_{k}=0, \pi / 6$, and $\pi / 4$, respectively. The left and right side figures show the real and imaginary part of $\Delta \boldsymbol{e}_{k}$, respectively. To show magnitude of the error vector, the one-tenth scaled true unit propagation vector $\widetilde{\boldsymbol{e}_{k}}$, which is a real number vector, is also depicted at the center of region in each figure.

Figure 4 Error dependencies on element size for the Even-Type configuration: Each figure in the left-hand side column shows the error at the center node of each side of the boundary. Figures in the right-hand side depict the error around corners. The difference among rows is the direction of the propagation vector, $\boldsymbol{k}$. The positions of the nodes to evaluate the error and the direction of $\boldsymbol{k}$ are depicted as a subfigure in the top right of each figure. Positions at the center of each side and at each corner are fixed but the nodes next to the corners are varying with change of $N$.

Figure 5 Comparison of error vectors in the Even-type and the Uneven-type configuration: The graph at top left side of each figure shows the error in whole region, while those at top right side and at bottom are zoomed vectors in the vicinity of the circled area in the figure of whole region. In the zoomed 
figures of Uneven-Type (b) the additional nodes are marked by diamond shaped symbols. All results are of $N=10, \phi_{k}=0$.

Figure 6 Error reduction around corners: $\quad(N=10, \phi=\pi / 6) \quad$ (a) Even sized elements: Sizes of all elements are equal. (b) Gradual element size variation: Each of two original elements connected to the corner node is replaced by 4 elements with a quarter size of the original element, respectively. Moreover, each of the elements adjacent to these is replaced by two elements of half size. (c) Fine resolution into 16 elements: Each of two original elements connected to the corner are, respectively, replaced by 8 elements of quarter size. 


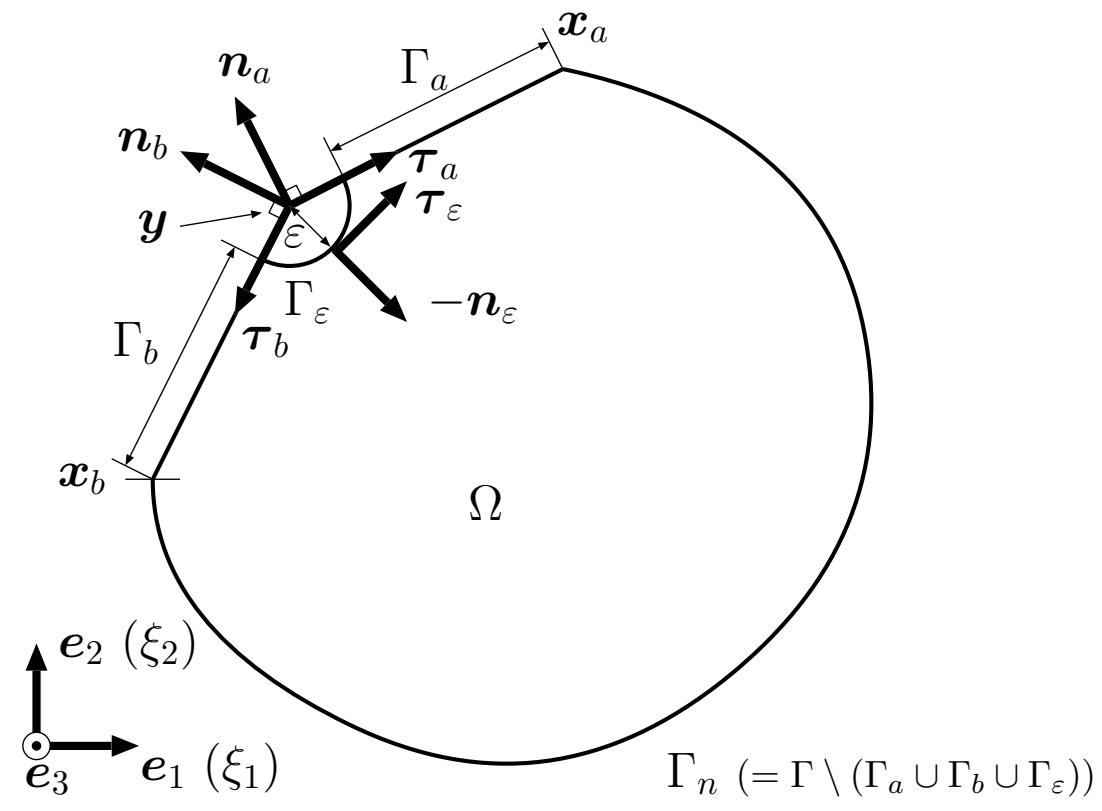

Figure 1: Boundary excluding the singular point. 


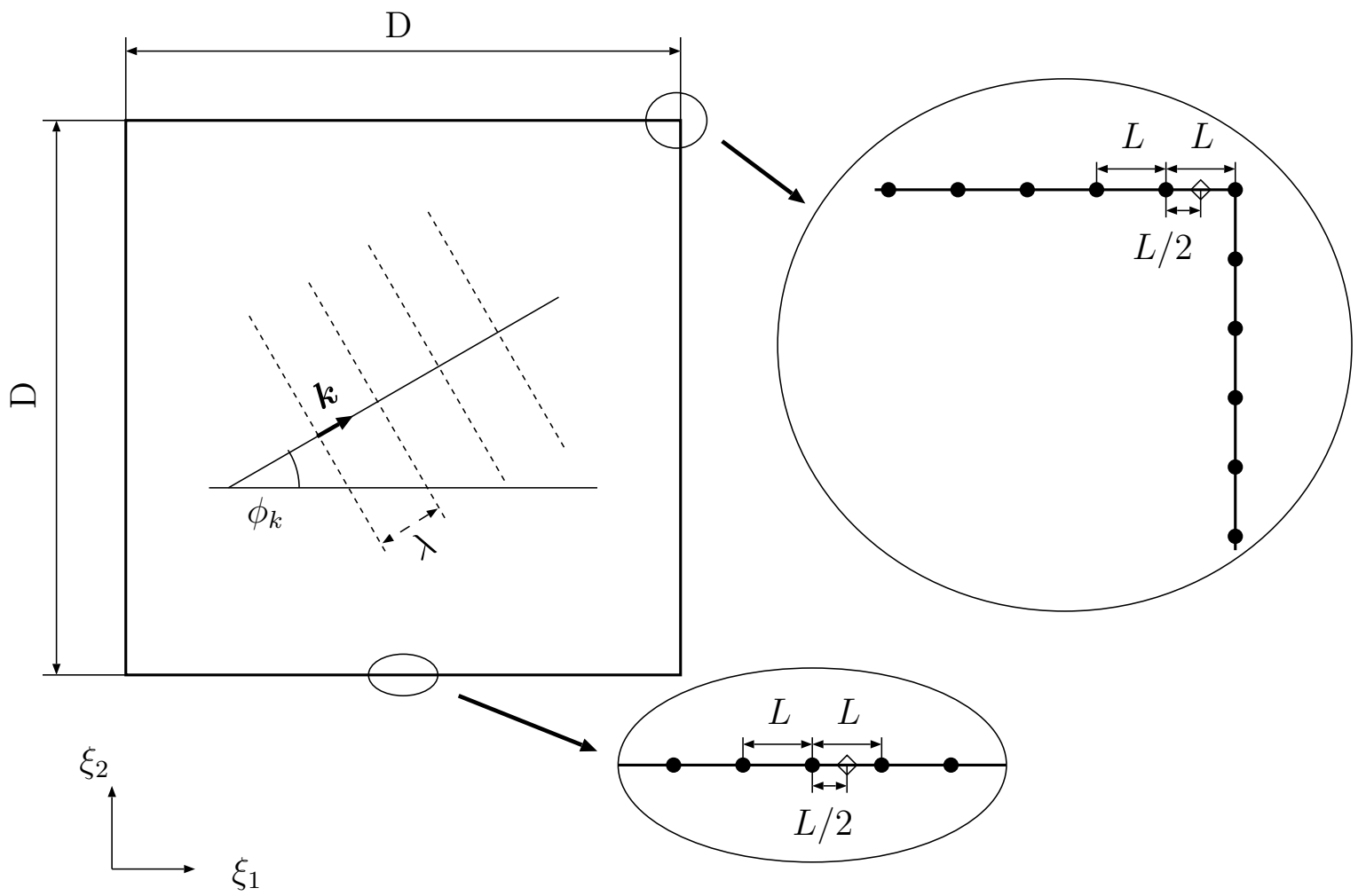

Figure 2: Node placements for Even-type and Uneven-type configurations: The filled circles show boundary nodes for the Even-type configuration. For the Uneven-type configuration, in which the interval of nodes are uneven, two additional nodes shown by diamond shaped symbols at the bottom side and around upper right corner are appended. The vector $\boldsymbol{k}$ shows the propagation vector. 

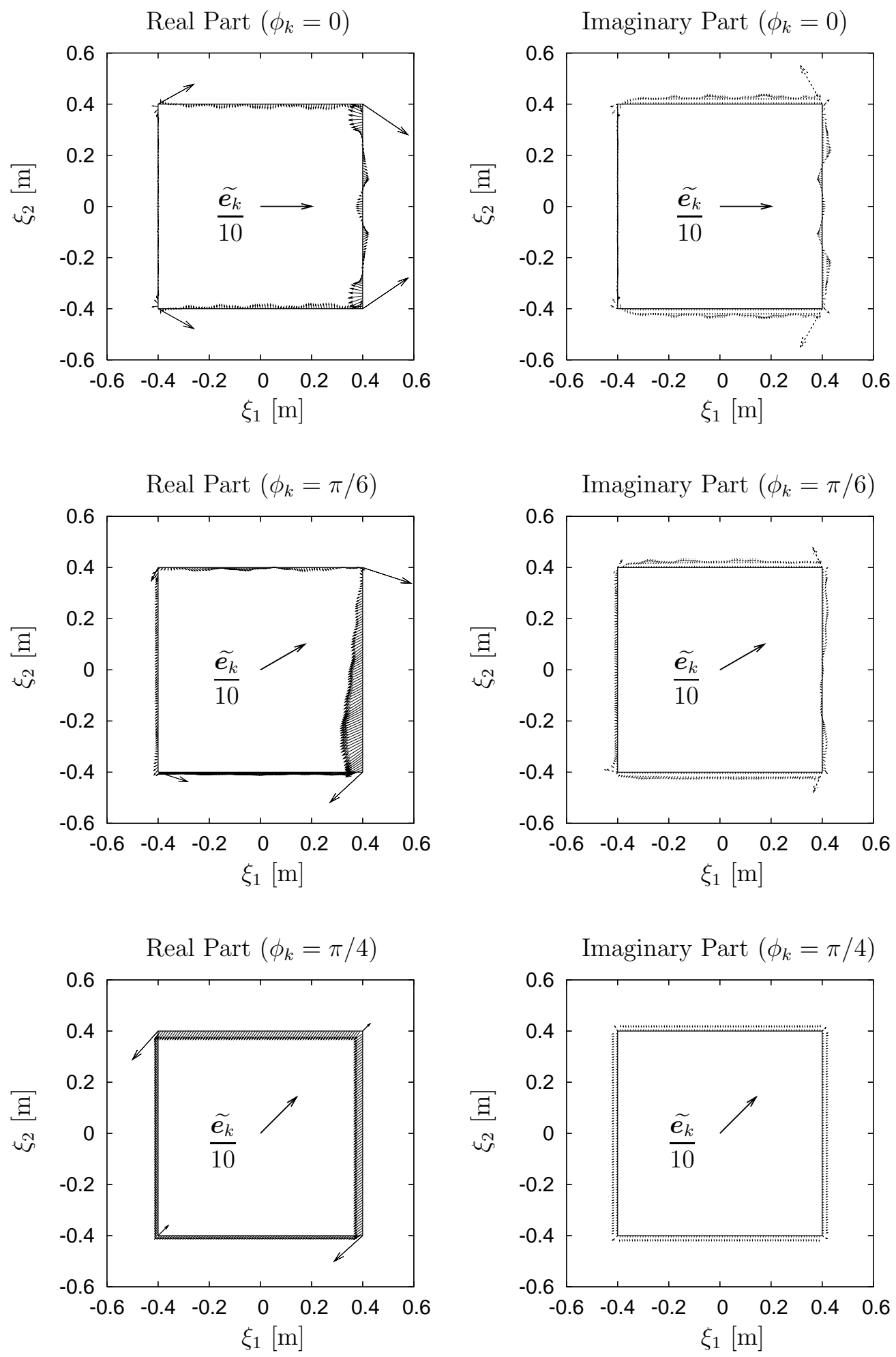

Figure 3: Complex error vector $\Delta \boldsymbol{e}_{k}$ of the Even-Type configuration $(N=10)$ : Each pair of figures in a row denotes a set of results for $\phi_{k}=0, \pi / 6$, and $\pi / 4$, respectively. The left and right side figures show the real and imaginary part of $\Delta \boldsymbol{e}_{k}$, respectively. To show magnitude of the error vector, the one-tenth scaled true unit propagation vector $\widetilde{\boldsymbol{e}_{k}}$, which is a real number vector, is also depicted at the center of region in each figure. 

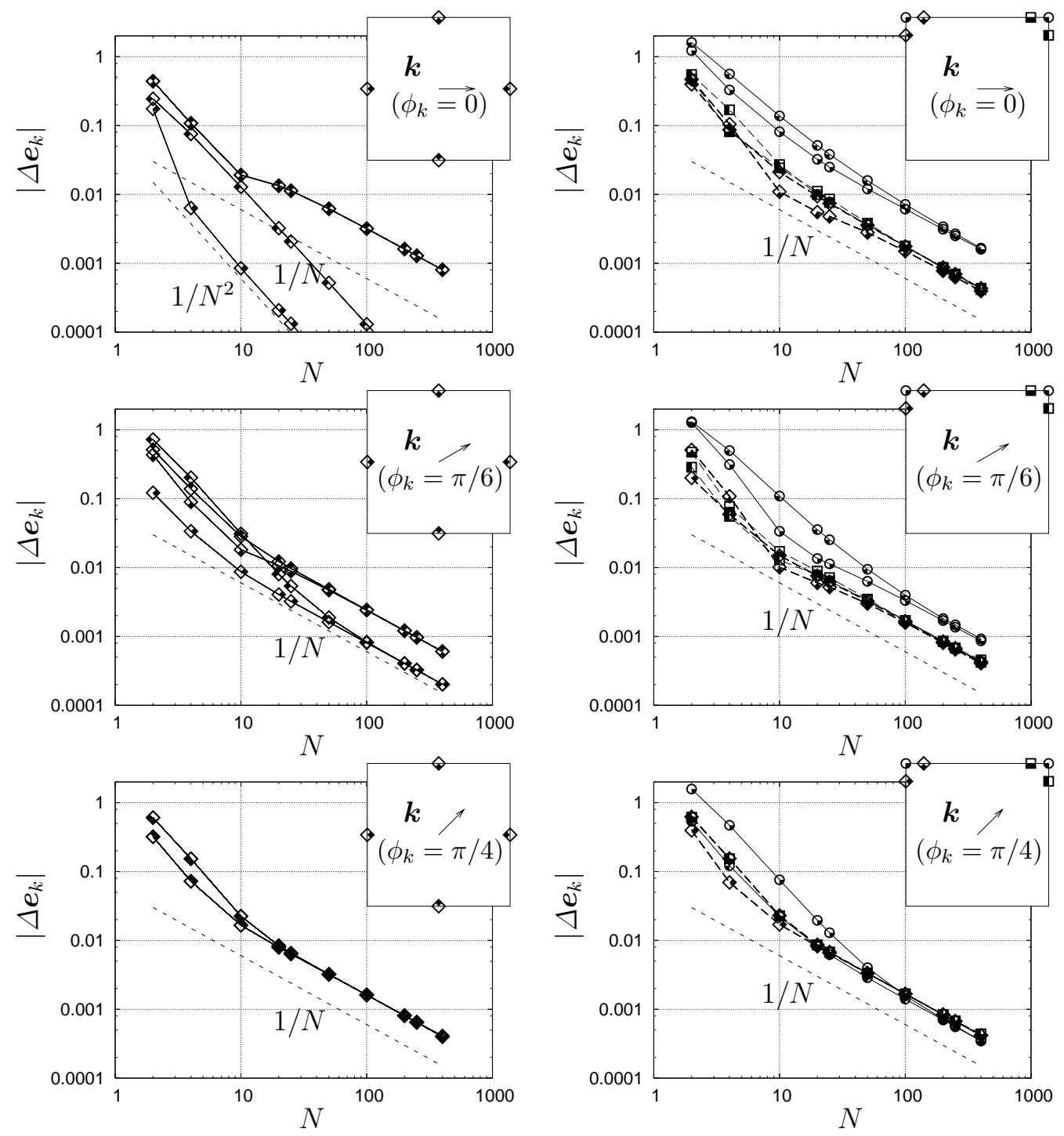

Figure 4: Error dependencies on element size for the Even-Type configuration: Each figure in the lefthand side column shows the error at the center node of each side of the boundary. Figures in the right-hand side depict the error around corners. The difference among rows is the direction of the propagation vector, $\boldsymbol{k}$. The positions of the nodes to evaluate the error and the direction of $\boldsymbol{k}$ are depicted as a subfigure in the top right of each figure. Positions at the center of each side and at each corner are fixed but the nodes next to the corners are varying with change of $N$. 


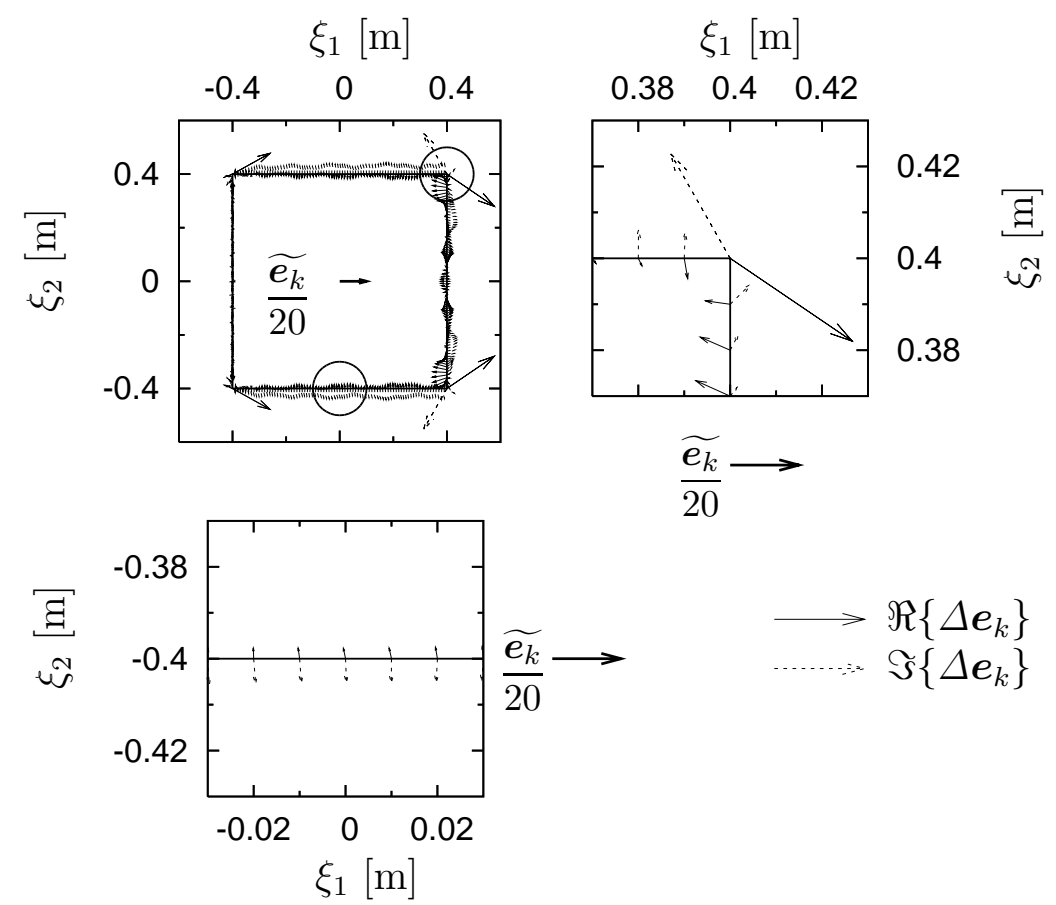

(a) Error vector $\Delta \boldsymbol{e}_{k}$ of Even-Type configuration
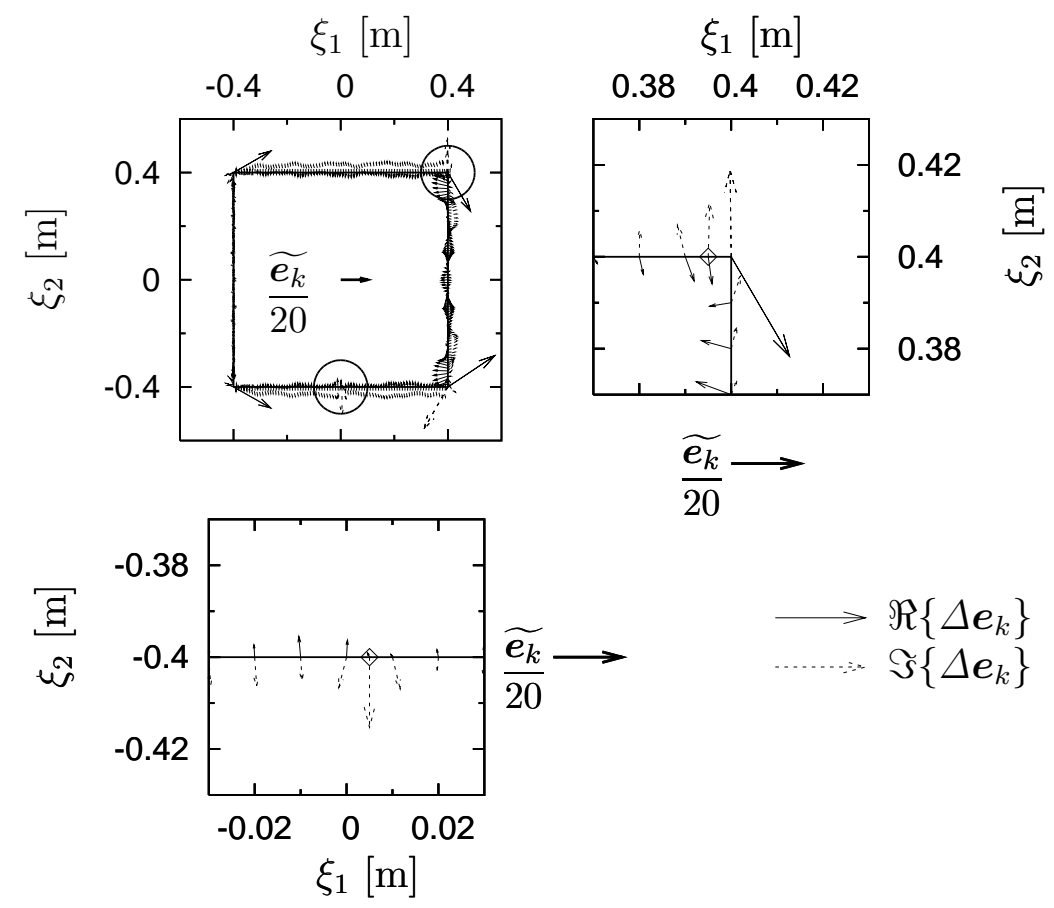

$\Im\left\{\Delta \boldsymbol{e}_{k}\right\}$

(b) Error vector $\Delta \boldsymbol{e}_{k}$ of Uneven-Type configuration

Figure 5: Comparison of error vectors in the Even-type and the Uneven-type configuration: The graph at top left side of each figure shows the error in whole region, while those at top right side and at bottom are zoomed vectors in the vicinity of the circled area in the figure of whole region. In the zoomed figures of Uneven-Type (b) the additional nodes are marked by diamond shaped symbols. All results are of $N=10$, $\phi_{k}=0$. 


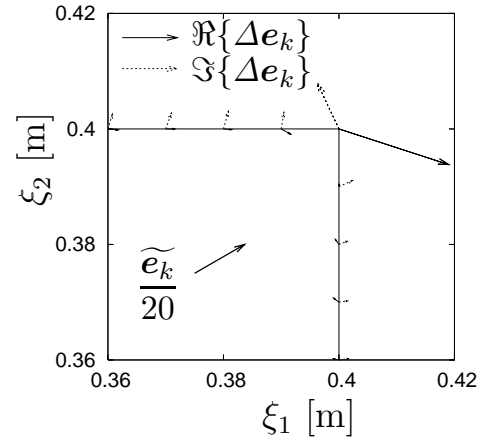

(a) Even sized element

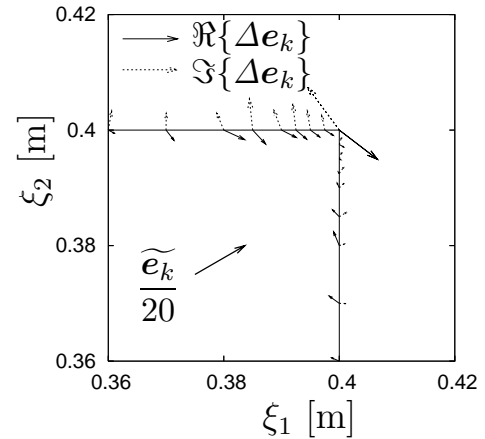

(b) Gradual element size variation

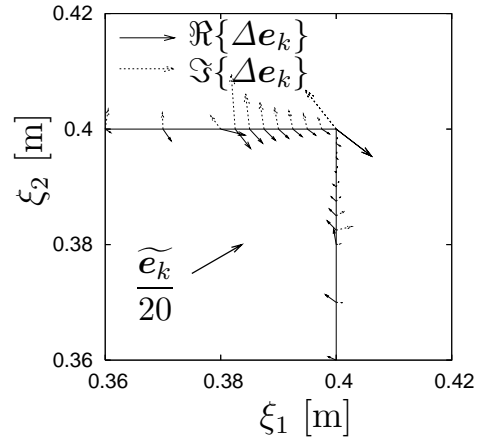

(c) Fine resolution into 16 elements

Figure 6: Error reduction around corners: $\quad(N=10, \phi=\pi / 6) \quad$ (a) Even sized elements: Sizes of all elements are equal. (b) Gradual element size variation: Each of two original elements connected to the corner node is replaced by 4 elements with a quarter size of the original element, respectively. Moreover, each of the elements adjacent to these is replaced by two elements of half size. (c) Fine resolution into 16 elements: Each of two original elements connected to the corner are, respectively, replaced by 8 elements of quarter size. 